\title{
Gravitational waves from the phase transition in the B-LSSM
}

\author{
Xing-Xing Dong, ${ }^{a, b}$ Tai-Fu Feng, ${ }^{a, b, c}$ Hai-Bin Zhang, ${ }^{a, b}$ Shu-Min Zhao ${ }^{a, b}$ \\ and Jin-Lei Yang ${ }^{d, e}$ \\ ${ }^{a}$ Department of Physics, Hebei University, \\ Baoding 071002, China \\ ${ }^{b}$ Key Laboratory of High-precision Computation and Application of Quantum Field Theory \\ of Hebei Province, Baoding, China \\ ${ }^{c}$ Department of Physics, Chongqing University, \\ Chongqing 401331, China \\ ${ }^{d}$ CAS Key Laboratory of Theoretical Physics, \\ Institute of Theoretical Physics, Chinese Academy of Sciences, \\ Beijing 100190, China \\ e School of Physical Sciences, University of Chinese Academy of Sciences, \\ Beijing 100049, China \\ E-mail: dxx_0304@163.com, fengtf@hbu.edu.cn, hbzhang@hbu.edu.cn, \\ zhaosm@hbu.edu.cn, yangjinlei@itp.ac.cn
}

ABSTRACT: Based on the gauge symmetry group $\mathrm{SU}(3)_{C} \otimes \mathrm{SU}(2)_{L} \otimes \mathrm{U}(1)_{Y} \otimes \mathrm{U}(1)_{B-L}$, the minimal supersymmetric extension of the SM with local B-L gauge symmetry(B-LSSM) has been introduced. In this model, we study the Higgs masses with the one-loop zero temperature effective potential corrections. Besides, the finite temperature effective potentials connected with two $\mathrm{U}(1)_{B-L}$ Higgs singlets are deduced specifically. Then we can obtain the gravitational wave spectrums generated from the strong first-order phase transition. In the B-LSSM, with the fine-tuned parameter regions, we can obtain the strength parameter $\alpha_{\theta} \sim 0.14$ and the ratio of speed to Hubble rate $\beta / H_{n} \sim 5$ at nucleation temperature, and then obtain observable gravitational wave signals. The gravitational wave signals can be as strong as $h^{2} \Omega_{G W} \sim 10^{-9}$, which may be detectable in the future experiments.

Keywords: Supersymmetry Phenomenology

ARXIV EPRINT: 2106.11084 


\section{Contents}

1 Introduction 1

2 The B-LSSM and the Higgs mass 3

2.1 The B-LSSM 3

2.2 Higgs mass in the B-LSSM 5

$\begin{array}{lll}2.3 & \text { Numerical discussion of the Higgs mass in the B-LSSM } & 7\end{array}$

3 Finite temperature effective potential in the B-LSSM $\quad 8$

3.1 Finite temperature effective potential 9

3.2 The PT from the finite temperature effective potential with the CP-even Higgs components $\phi_{\eta}$ and $\phi_{\bar{\eta}}$

4 GW generation by cosmological first-order PT 12

4.1 Scalar potential parameters related to the GW spectrum 12

$\begin{array}{lll}4.2 \text { GW spectrum } & 13\end{array}$

$\begin{array}{lll}\text { 4.2.1 Bubble collisions } & 15\end{array}$

$\begin{array}{lll}4.2 .2 & \text { Sound waves } & 15\end{array}$

$\begin{array}{lll}4.2 .3 & \text { Turbulence } & 16\end{array}$

5 The numerical results of GW spectrum in the B-LSSM 16

$\begin{array}{llr}6 & \text { Conclusion } & 19\end{array}$

$\begin{array}{ll}\text { A The anomaly free of B-LSSM } & 20\end{array}$

$\begin{array}{ll}\text { B The vacuum of Higgs doublets and Higgs singlets } & 21\end{array}$

$\begin{array}{ll}\text { C The value of parameter } g_{Y B} & 22\end{array}$

$\begin{array}{ll}\text { D The neutralino mass matrix } & 23\end{array}$

E The one-loop correction $A_{\text {one-loop }} \quad 23$

\section{Introduction}

Based on the gauge group $\mathrm{SU}(3)_{C} \otimes \mathrm{SU}(2)_{L} \otimes \mathrm{U}(1)_{Y}$, the standard model(SM) has been successfully established. Nevertheless, the SM possesses some limitation and additional $\mathrm{U}(1)_{B-L}$ gauge interaction will be a promising extension to the SM. Therefore, the minimal supersymmetric extension of the SM(MSSM) [1-4] with local B-L gauge symmetry (B-LSSM) is introduced, where $B$ represents baryon number and $L$ stands for lepton number [5-9]. In this model, the invariance under $\mathrm{U}(1)_{B-L}$ gauge group imposes the R-parity 
conservation to avoid proton decay [10]. Besides, the right-handed neutrino superfields have been imported in the B-LSSM to obtain the tiny neutrino masses through type-I seesaw mechanism. Thus, B-LSSM provides an elegant solution to the existence of the light left-handed neutrino. Then the possibility of baryogenesis via leptogenesis will be well explained. Furthermore, due to the introduction of the additional singlet Higgs states and right-handed (s)neutrinos, B-LSSM alleviates the hierarchy problem through additional parameter space released from the LEP, Tevatron and LHC constraints [11, 12]. Other than this, the model can also provide much more dark matter candidates [13-16] than the MSSM.

The gravitational wave(GW) signals have been detected at the Laser Interferometer Gravitational Wave Observer (LIGO) [17, 18], which urges physicists to explore the various universe mysteries. The sources of GW signals can be sensitive to arise from the firstorder phase transition(PT) in the early universe [19-23]. The symmetric broken phase is produced in the form of bubbles. When the universe cools down to the nucleation temperature, bubbles of broken phase may nucleate in the background of symmetric phase. Bubbles expand, collide, merge and finally fill the whole universe to finish the first-order PT. Then, the stochastic GW signals can be generated through the bubble collisions, sound waves after the bubble collision and magnetohydrodynamic turbulent of the surrounding plasma $[22,24,25]$.

Unfortunately, the electroweak PT in the SM turns out to be too weak to obtain GW signals [26-28]. In order to realize strong first-order PT and finally obtain the GW spectrums, physicists have studied various extensions of the SM, such as the model with a dimension-six operator [29-31], NMSSM [32-34] and other studies [35-37]. In this paper, we hope to investigate the GW spectrums produced from the first-order PT of the B-LSSM. We find that the strength of GW signals will be up to $h^{2} \Omega_{G W} \sim 10^{-9}$, which can be detected by the future experiments, such as the LISA with N2A5M5L6 design configurations, the Big Bang Observer(BBO), DECi-hertz Interferometer Observatory(DECIGO) and UltimateDECIGO [38, 39].

In this paper, after discussing the Higgs mass, we mainly study the GW spectrums generated from the strong first-order PT of the B-LSSM. We first introduce characteristics of the B-LSSM, then we discuss the Higgs masses corrected by the one-loop zero temperature effective potential in section II. In section III, we compute the concrete finite temperature effective potential associated with the CP-even Higgs components $\phi_{\eta}$ and $\phi_{\bar{\eta}}$. Meanwhile, we derive the GW generation by cosmological first-order PT in section IV. The GW spectrums are comprised by the bubble collision, sound wave and turbulence contributions. The numerical results of the GW spectrums that depend on the model parameters will be further illustrated in section V. Last but not least, we summarize the conclusion in section VI. The anomaly free of B-LSSM will be demonstrated in appendix A. The vacuum of eq.2 is indeed the global one and there is no charge breaking nor CP violating deeper minima, which will be demonstrated in appendix B. The value of parameter $g_{Y B}$ will be discussed in appendix C. The neutralino mass matrix will be searched in appendix D. The one-loop correction through the one-loop zero temperature effective potential $A_{\text {one-loop }} \equiv \frac{B_{\eta}+\Delta_{B_{\eta}}}{\sqrt{\left(m_{\eta}^{2}+\mu_{\eta}^{2}+\Delta_{11}\right)\left(m_{\bar{\eta}}^{2}+\mu_{\eta}^{2}+\Delta_{22}\right)}}$ will be deduced in appendix E. 


\section{The B-LSSM and the Higgs mass}

\subsection{The B-LSSM}

In 1967, Sakharov proposed three necessary conditions for dynamics to produce asymmetry between matter and anti-matter in the universe [40]: (1) baryon number(B) nonconservation; (2) charge conjugation $(\mathrm{C})$ transformation and charge conjugate-parity $(\mathrm{CP})$ joint transformation non-conservation; (3) The system deviation from thermal equilibrium. Through a lot of research, people realize that the SM can satisfy Sakharov's three conditions. However, the quantitative calculation shows that the CP violation in CKM matrix is very small and far from producing the observed matter-antimatter asymmetry in the present SM. The literature [41] has pointed out that the CP violation from the CKM matrix only could be sufficient in models where the SM Yukawa couplings are field-dependent and evolve during the electroweak PT. In addition, in order to produce strong first-order electroweak PT, the mass of Higgs particle in the SM must be less than $66 \mathrm{GeV}[42-$ 44]. We study the ref. [45], and find a lower bound of the mass of the SM Higgs boson $m_{h}^{0}>114.4 \mathrm{GeV}$ by the experimental LEP II. So the possibility of a first-order electroweak PT within the SM has been ruled out by Higgs searches at the LEP experiment. In 2012, the mass of physical Higgs particle was found to be around $125 \mathrm{GeV}$ by the European Large Hadron Collider (LHC) [46, 47], which directly denied the possibility of realizing the electroweak baryon number production mechanism (baryogenesis) in the SM. Therefore, the current SM can not explain the matter and anti-matter asymmetries in the universe.

In any models with a non-anomalous global $\mathrm{U}(1)_{B-L}$ symmetry, which includes the BLSSM we studied, the baryon number and lepton number are broken by Sphaleron process respectively, but the difference between the two is conserved. This links the change of the baryon number with the change of the lepton number, and the asymmetry of the baryon number can be converted from the asymmetry of the lepton number through the sphaleron process. In general, the mechanism of lepton number asymmetry (leptogenesis) requires the lepton number violating process, the $\mathrm{C}$ and $\mathrm{CP}$ destruction of the lepton part and the realization of the non-equilibrium state. These conditions can be achieved in the general models within the mass neutrino. The B-LSSM introduces the right-handed neutrinos. Besides, neutrinos are Majorana type and gain mass through the type-I seesaw mechanism, which breaks the lepton number symmetry. In B-LSSM, the C or CP symmetry is broken. In addition, the decoupling of heavy right-handed neutrinos provides nonequilibrium conditions. Sphhaleron process only acts directly on left-handed fermions, and can partially convert the lepton number of left-handed leptons to the baryon number, thus explains the baryon number asymmetry. Therefore, the possibility of baryogenesis via leptogenesis can be realized within the B-LSSM.

In the B-LSSM, the local gauge group is defined as $\mathrm{SU}(3)_{C} \otimes \mathrm{SU}(2)_{L} \otimes \mathrm{U}(1)_{Y} \otimes \mathrm{U}(1)_{B-L}$. The specification of the quantum numbers of fields in the B-LSSM will be discussed in the table 1. Besides, B-LSSM is assumed the gauged one, and we have proved carefully that this model is anomaly free. The concrete demonstrations will be given out in the appendix A. BLSSM introduces $\mathrm{U}(1)_{B-L}$ gauge field, right-handed neutrinos $\nu_{i}^{c}$ and their superpartners. The masses of right-handed neutrinos will be constructed by $Y_{x, i j} \hat{\nu}_{i}^{c} \hat{\eta} \hat{\nu}_{j}^{c}$. Then we can 


\begin{tabular}{|c|c|c|c|c|}
\hline $\mathrm{SF}$ & Spin 0 & Spin $\frac{1}{2}$ & Generations & $\left(\mathrm{U}(1)_{Y} \otimes \mathrm{SU}(2) \otimes \mathrm{SU}(3) \otimes \mathrm{U}(1)_{B-L}\right)$ \\
\hline$\hat{H}_{d}$ & $H_{d}$ & $\tilde{H}_{d}$ & 1 & $\left(-\frac{1}{2}, \mathbf{2}, \mathbf{1}, 0\right)$ \\
$\hat{H}_{u}$ & $H_{u}$ & $\tilde{H}_{u}$ & 1 & $\left(\frac{1}{2}, \mathbf{2}, \mathbf{1}, 0\right)$ \\
$\hat{Q}$ & $\tilde{Q}$ & $Q$ & 3 & $\left(\frac{1}{6}, \mathbf{2}, \mathbf{3}, \frac{1}{6}\right)$ \\
$\hat{L}$ & $\tilde{L}$ & $L$ & 3 & $\left(-\frac{1}{2}, \mathbf{2}, \mathbf{1},-\frac{1}{2}\right)$ \\
$\hat{D}$ & $\tilde{D}_{R}^{*}$ & $D_{R}^{*}$ & 3 & $\left(\frac{1}{3}, \mathbf{1}, \overline{\mathbf{3}},-\frac{1}{6}\right)$ \\
$\hat{U}$ & $\tilde{U}_{R}^{*}$ & $U_{R}^{*}$ & 3 & $\left(-\frac{2}{3}, \mathbf{1}, \overline{\mathbf{3}},-\frac{1}{6}\right)$ \\
$\hat{R}$ & $\tilde{R}^{*}$ & $R^{*}$ & 3 & $\left(1, \mathbf{1}, \mathbf{1}, \frac{1}{2}\right)$ \\
$\hat{\nu}$ & $\tilde{\nu}_{R}^{*}$ & $\nu_{R}^{*}$ & 3 & $\left(0, \mathbf{1}, \mathbf{1}, \frac{1}{2}\right)$ \\
$\hat{\eta}$ & $\eta$ & $\tilde{\eta}$ & 1 & $(0, \mathbf{1}, \mathbf{1},-1)$ \\
$\hat{\bar{\eta}}$ & $\bar{\eta}$ & $\tilde{\bar{\eta}}$ & 1 & $(0, \mathbf{1}, \mathbf{1}, 1)$ \\
\hline
\end{tabular}

Table 1. The quantum numbers of fields in the B-LSSM.

obtain the tiny neutrinos masses through the type-I seesaw mechanism after the righthanded neutrinos and left-handed neutrinos mixing together. The B-LSSM superpotential is deduced as

$$
W_{B-L}=\mathcal{W}_{M S S M}-\mu_{\eta} \hat{\eta} \hat{\bar{\eta}}+Y_{x, i j} \hat{\nu}_{i}^{c} \hat{\eta} \hat{\nu}_{j}^{c}+Y_{\nu, i j} \hat{L}_{i} \hat{H}_{u} \hat{\nu}_{j}^{c}
$$

where $\mathcal{W}_{M S S M}$ is the superpotential of MSSM. $i, j$ represent the generation indices, $Y_{x, i j}$ and $Y_{\nu, i j}$ correspond to the Yukawa coupling coefficients. $\mu_{\eta}$, considered as a parameter with mass dimension, is the supersymmetric mass between $\mathrm{U}(1)_{B-L}$ Higgs singlets $\eta$ and $\bar{\eta}$.

In the B-LSSM, the Higgs doublets and Higgs singlets obtain the nonzero vacuum expectation values, which break the gauge group $\mathrm{SU}(3) \otimes \mathrm{U}(1)_{Y} \otimes \mathrm{U}(1)_{B-L}$ down to $\mathrm{U}(1)_{e m}$ at zero temperature. For the model's parameters values considered in our analyses, the vacuum of eq. 2.2 is indeed the global one and that there is no charge breaking nor $\mathrm{CP}$ violating deeper minima. The concrete discussion will be shown in appendix B.

$$
\begin{aligned}
H_{d}^{0} & =\frac{1}{\sqrt{2}}\left(\phi_{d}+v_{d}+i \sigma_{d}\right), & H_{u}^{0} & =\frac{1}{\sqrt{2}}\left(\phi_{u}+v_{u}+i \sigma_{u}\right), \\
\eta & =\frac{1}{\sqrt{2}}\left(\phi_{\eta}+v_{\eta}+i \sigma_{\eta}\right), & \bar{\eta} & =\frac{1}{\sqrt{2}}\left(\phi_{\bar{\eta}}+v_{\bar{\eta}}+i \sigma_{\bar{\eta}}\right),
\end{aligned}
$$

where $\phi_{d}, \phi_{u}, \phi_{\eta}, \phi_{\bar{\eta}}$ represent the CP-even Higgs components and $\sigma_{d}, \sigma_{u}, \sigma_{\eta}, \sigma_{\bar{\eta}}$ correspond to the CP-odd Higgs components. The VEVs of the $\mathrm{U}(1)_{B-L}$ Higgs singlets $\hat{\eta}$ and $\hat{\bar{\eta}}$ satisfy $u=\sqrt{v_{\eta}^{2}+v_{\bar{\eta}}^{2}}$. While the VEVs of the Higgs doublets $\hat{H}_{d}$ and $\hat{H}_{u}$ are $v_{d}$ and $v_{u}$, which satisfy $v=\sqrt{v_{d}^{2}+v_{u}^{2}}$. We take $t b^{\prime}=\frac{v_{\bar{\eta}}}{v_{\eta}}$ by analogy to the definition $t b=\frac{v_{u}}{v_{d}}$ in the MSSM. 


\subsection{Higgs mass in the B-LSSM}

First, in the base $\left(\phi_{d}, \phi_{u}, \phi_{\eta}, \phi_{\bar{\eta}}\right)$, the tree level mass squared matrix for neutral CP-even scalars $M_{h}^{2}$ is deduced as:

$$
M_{h}^{2}=u^{2} \times\left(\begin{array}{cccc}
\frac{1}{4} \frac{g^{2} x^{2}}{1+t b^{2}}+n^{2} t b & -\frac{1}{4} g^{2} \frac{x^{2} t b}{1+t b^{2}}-n^{2} & \frac{1}{2} g_{B} g_{Y B} \frac{x}{T} & -\frac{1}{2} g_{B} g_{Y B} \frac{x \times t b^{\prime}}{T} \\
-\frac{1}{4} g^{2} \frac{x^{2} t b}{1+t b^{2}}-n^{2} & \frac{1}{4} \frac{g^{2} x^{2} t b^{2}}{1+t b^{2}}+\frac{n^{2}}{t b} & \frac{1}{2} g_{B} g_{Y B} \frac{x \times t b}{T} & \frac{1}{2} g_{B} g_{Y B} \frac{x \times t b \times t b^{\prime}}{T} \\
\frac{1}{2} g_{B} g_{Y B} \frac{x}{T} & \frac{1}{2} g_{B} g_{Y B} \frac{x \times t b}{T} & \frac{g_{B}^{2}}{1+t b^{\prime 2}}+t b^{\prime} N^{2} & -g_{B}^{2} \frac{t b^{\prime}}{1+t b^{\prime 2}}-N^{2} \\
-\frac{1}{2} g_{B} g_{Y B} \frac{x \times t b^{\prime}}{T} & \frac{1}{2} g_{B} g_{Y B} \frac{x \times t b \times t b^{\prime}}{T} & -g_{B}^{2} \frac{t b^{\prime}}{1+t b^{\prime 2}}-N^{2} & g_{B}^{2} \frac{t b^{\prime 2}}{1+t b^{\prime 2}}+\frac{N^{2}}{t b^{\prime}}
\end{array}\right) .
$$

Here, $g^{2}=g_{1}^{2}+g_{2}^{2}+g_{Y B}^{2}, T=\sqrt{1+t b^{2}} \sqrt{1+t b^{\prime 2}}, n^{2}=\frac{\operatorname{Re}(B \mu)}{u^{2}}$ and $N^{2}=\frac{\operatorname{Re}\left(B_{\eta}\right)}{u^{2}}$.

Then, we will consider the radiative corrections $\Delta \Pi$ from the one-loop zero temperature effective potential to the tree level Higgs mass squared matrix.

$$
m_{h}^{2}=M_{h}^{2}+\Delta \Pi, \quad \Delta \Pi=\left(\begin{array}{cccc}
\Delta \Pi_{11} & \Delta \Pi_{12} & \Delta \Pi_{13} & \Delta \Pi_{14} \\
\Delta \Pi_{21} & \Delta \Pi_{22} & \Delta \Pi_{23} & \Delta \Pi_{24} \\
\Delta \Pi_{31} & \Delta \Pi_{32} & \Delta \Pi_{33} & \Delta \Pi_{34} \\
\Delta \Pi_{41} & \Delta \Pi_{42} & \Delta \Pi_{43} & \Delta \Pi_{44}
\end{array}\right)
$$

with $\Delta \Pi_{i i}=\left[-\frac{1}{\phi_{i}} \frac{\partial \Delta V_{1}}{\partial \phi_{i}}+\frac{\partial^{2} \Delta V_{1}}{\partial \phi_{i}^{2}}\right]_{\phi_{i}=v_{i}}$ and $\Delta \Pi_{i j}=\left[\frac{\partial^{2} \Delta V_{1}}{\partial \phi_{i} \partial \phi_{j}}\right]_{\phi_{i}=v_{i}, \phi_{j}=v_{j}}, v_{i}, v_{j} \in\left(v_{d}, v_{u}, v_{\eta}, v_{\bar{\eta}}\right)$ and $\phi_{i, j}$ are the CP-even Higgs components $\phi_{d}, \phi_{u}, \phi_{\eta}, \phi_{\bar{\eta}} . \Delta V_{1}$ represents the one-loop zero temperature effective potential whose full form has be discussed in the literature [48]. In principle, the radiative correction is dominated by the contributions of top quark, bottom quark, stop quarks and sbottom quarks.

$$
\begin{aligned}
\Delta V_{1}= & -\frac{3}{(4 \pi)^{2}}\left[\frac{m_{t}^{4}}{4}\left(\ln \left(\frac{m_{t}^{2}}{\Lambda^{2}}\right)-\frac{3}{2}\right)-\frac{1}{2} \frac{m_{\tilde{t}}^{4}}{4}\left(\ln \left(\frac{m_{\tilde{t}}^{2}}{\Lambda^{2}}\right)-\frac{3}{2}\right)\right] \\
& -\frac{3}{(4 \pi)^{2}}\left[\frac{m_{b}^{4}}{4}\left(\ln \left(\frac{m_{b}^{2}}{\Lambda^{2}}\right)-\frac{3}{2}\right)-\frac{1}{2} \frac{m_{\tilde{b}}^{4}}{4}\left(\ln \left(\frac{m_{\tilde{b}}^{2}}{\Lambda^{2}}\right)-\frac{3}{2}\right)\right] .
\end{aligned}
$$

Here, the masses of top quark and bottom quark are respectively $m_{t}=\frac{1}{\sqrt{2}} Y_{t} \phi_{u}$ and $m_{b}=$ $\frac{1}{\sqrt{2}} Y_{b} \phi_{d}$. Additionally, the mass squared matrix of stop quark and sbottom quark will be deduced respectively in the basis $\left(\tilde{U}_{L}, \tilde{U}_{R}\right)$ and $\left(\tilde{D}_{L}, \tilde{D}_{R}\right)$.

$$
m_{\tilde{t}}^{2}=\left(\begin{array}{cc}
m_{\tilde{t}_{L}}^{2} & m_{\tilde{t}_{L R}}^{2} \\
m_{\tilde{t}_{R L}}^{2} & m_{\tilde{t}_{R}}^{2}
\end{array}\right), \quad m_{\tilde{b}}^{2}=\left(\begin{array}{cc}
m_{\tilde{b}_{L}}^{2} & m_{\tilde{b}_{L R}}^{2} \\
m_{\tilde{b}_{R L}}^{2} & m_{\tilde{b}_{R}}^{2}
\end{array}\right)
$$


where,

$$
\begin{aligned}
& m_{\tilde{t}_{L}}^{2}=+\frac{1}{24}[ 3 g_{2}^{2}\left(-\phi_{u}^{2}+\phi_{d}^{2}\right)+\left(g_{1}^{2}+g_{Y B}^{2}\right)\left(-\phi_{d}^{2}+\phi_{u}^{2}\right)-2 g_{B}^{2}\left(-\phi_{\bar{\eta}}^{2}+\phi_{\eta}^{2}\right) \\
&\left.+g_{Y B} g_{B}\left(2 \phi_{\bar{\eta}}^{2}-2 \phi_{\eta}^{2}-\phi_{d}^{2}+\phi_{u}^{2}\right)\right]+\frac{1}{2}\left(2 m_{\tilde{Q}_{3}}^{2}+\phi_{u}^{2} Y_{t}^{\dagger} Y_{t}\right), \\
& m_{\tilde{t}_{R}}^{2}=+\frac{1}{24}[ 2 g_{B}^{2}\left(-\phi_{\bar{\eta}}^{2}+\phi_{\eta}^{2}\right)+4\left(g_{1}^{2}+g_{Y B}^{2}\right)\left(-\phi_{u}^{2}+\phi_{d}^{2}\right) \\
&\left.+g_{Y B} g_{B}\left(-8 \phi_{\bar{\eta}}^{2}+8 \phi_{\eta}^{2}-\phi_{u}^{2}+\phi_{d}^{2}\right)\right]+\frac{1}{2}\left(2 m_{\tilde{U}_{3}}^{2}+\phi_{u}^{2} Y_{t} Y_{t}^{\dagger}\right), \\
& m_{\tilde{t}_{R L}}^{2}=\left(m_{\tilde{t}_{L R}}^{2}\right)^{\dagger}=\frac{1}{\sqrt{2}}\left(\phi_{u} T_{t}-\phi_{d} \mu^{*} Y_{t)} .\right.
\end{aligned}
$$

Similarly, the elements in the sbottom mass squared matrix are

$$
\begin{aligned}
& m_{\tilde{b}_{L}}^{2}=+\frac{1}{24}[-2 g_{B}^{2}\left(-\phi_{\bar{\eta}}^{2}+\phi_{\eta}^{2}\right)+\left(3 g_{2}^{2}+g_{1}^{2}+g_{Y B}^{2}\right)\left(-\phi_{d}^{2}+\phi_{u}^{2}\right) \\
&\left.+g_{Y B} g_{B}\left(2 \phi_{\bar{\eta}}^{2}-2 \phi_{\eta}^{2}-\phi_{d}^{2}+\phi_{u}^{2}\right)\right]+\frac{1}{2}\left(2 m_{\tilde{Q}_{3}}^{2}+\phi_{d}^{2} Y_{b}^{\dagger} Y_{b}\right), \\
& m_{\tilde{b}_{R}}^{2}=+\frac{1}{24}\left[2\left(\left(g_{1}^{2}+g_{Y B}^{2}\right)\left(-\phi_{d}^{2}+\phi_{u}^{2}\right)+g_{B}^{2}\left(-\phi_{\bar{\eta}}^{2}+\phi_{\eta}^{2}\right)\right)\right. \\
&\left.+g_{Y B} g_{B}\left(4 \phi_{\bar{\eta}}^{2}-4 \phi_{\eta}^{2}-\phi_{u}^{2}+\phi_{d}^{2}\right)\right]+\frac{1}{2}\left(2 m_{\tilde{D}_{3}}^{2}+\phi_{d}^{2} Y_{b} Y_{b}^{\dagger}\right), \\
& m_{\tilde{b}_{R L}}^{2}=\left(m_{\tilde{b}_{L R}}^{2}\right)^{\dagger}=\frac{1}{\sqrt{2}}\left(\phi_{d} T_{b}-\phi_{u} Y_{b} \mu^{*}\right) .
\end{aligned}
$$

Then, the mass eigenvalues of stop and sbottom quarks will be given by

$$
\begin{aligned}
& m_{\tilde{t}_{1,2}}^{2}=\frac{1}{2}\left(m_{\tilde{t}_{L}}^{2}+m_{\tilde{t}_{R}}^{2}\right) \pm \frac{1}{2}\left[\left(m_{\tilde{t}_{L}}^{2}-m_{\tilde{t}_{R}}^{2}\right)^{2}+4 m_{\tilde{t}_{L R}}^{2} m_{\tilde{t}_{R L}}^{2}\right]^{\frac{1}{2}} \\
& m_{\tilde{b}_{1,2}}^{2}=\frac{1}{2}\left(m_{\tilde{b}_{L}}^{2}+m_{\tilde{b}_{R}}^{2}\right) \pm \frac{1}{2}\left[\left(m_{\tilde{b}_{L}}^{2}-m_{\tilde{b}_{R}}^{2}\right)^{2}+4 m_{\tilde{b}_{L R}}^{2} m_{\tilde{b}_{R L}}^{2}\right]^{\frac{1}{2}}
\end{aligned}
$$

Therefore, the radiative corrections $\Delta \Pi$ from the one-loop zero temperature effective potential will be deduced as:

$$
\begin{aligned}
\Delta \Pi_{i i}= & \frac{3}{(4 \pi)^{2}}\left[\sum_{k=t, b}\left(\frac{2}{\phi_{i}} f\left(m_{k}^{2}\right) \frac{\partial m_{k}^{2}}{\partial \phi_{i}}-2 f\left(m_{k}^{2}\right) \frac{\partial^{2} m_{k}^{2}}{\partial \phi_{i}^{2}}-\frac{1}{2} \ln \left(\frac{m_{k}^{2}}{\Lambda^{2}}\right) \frac{\partial m_{k}^{2}}{\partial \phi_{i}} \frac{\partial m_{k}^{2}}{\partial \phi_{i}}\right)\right. \\
& \left.+\sum_{k=\tilde{t}_{1}, \tilde{t}_{2}, \tilde{b}_{1}, \tilde{b}_{2}}\left(-\frac{1}{\phi_{i}} f\left(m_{k}^{2}\right) \frac{\partial m_{k}^{2}}{\partial \phi_{i}}+f\left(m_{k}^{2}\right) \frac{\partial^{2} m_{k}^{2}}{\partial \phi_{i}^{2}}+\frac{1}{4} \ln \left(\frac{m_{k}^{2}}{\Lambda^{2}}\right) \frac{\partial m_{k}^{2}}{\partial \phi_{i}} \frac{\partial m_{k}^{2}}{\partial \phi_{i}}\right)\right], \\
\Delta \Pi_{i j}= & \frac{3}{(4 \pi)^{2}} \sum_{k=\tilde{t}_{1}, \tilde{t}_{2}, \tilde{b}_{1}, \tilde{b}_{2}}\left(f\left(m_{k}^{2}\right) \frac{\partial^{2} m_{k}^{2}}{\partial \phi_{i} \partial \phi_{j}}+\frac{1}{4} \ln \left(\frac{m_{k}^{2}}{\Lambda^{2}}\right) \frac{\partial m_{k}^{2}}{\partial \phi_{i}} \frac{\partial m_{k}^{2}}{\partial \phi_{j}}\right) .
\end{aligned}
$$

Here, $f\left(m_{k}^{2}\right)=\frac{1}{4} m_{k}^{2}\left[\ln \left(\frac{m_{k}^{2}}{\Lambda^{2}}\right)-1\right], \Lambda$ is the renormalization scale and we take $\Lambda=1 \mathrm{TeV}$ in the following numerical discussion. $m_{k}$ represent the corresponding particle masses. The square matrix $m_{h}^{2}$ will be diagonalized to the mass eigenstate by the unitary matrix $Z_{h_{i}}$. 


\subsection{Numerical discussion of the Higgs mass in the B-LSSM}

The mass of physical Higgs boson reads $m_{h^{0}}=125.1 \pm 0.14 \mathrm{GeV}$ by the latest LHC experiments [49]. We consider the impact of LHC constraints from the light top and bottom squarks. Limits are considered in the supersymmetric models at the $95 \%$ confidence level, and bottom squarks with mass up to $1.5 \mathrm{TeV}$ are excluded [50]. The results are interpreted in simplified models featuring direct production of the light top squark, excluding light top squark masses up to about $1.22 \mathrm{TeV}$ at $95 \%$ confidence level [51]. In our recently published article [52], we had studied the CP-even Higgs boson mass through the finetuning in both the MSSM and B-LSSM. We found the fine-tuning measure was $\Delta_{F T} \gtrsim$ $35\left(\lesssim 2.9 \%\right.$ fine tuning) according to $m_{\tilde{t}_{1}} \gtrsim 1.5 \mathrm{TeV}$ in the MSSM. However, the fine-tuning measure in the B-LSSM was $\Delta_{F T} \gtrsim 22\left(\lesssim 4.5 \%\right.$ fine tuning) according to $m_{\tilde{t}_{1}} \gtrsim 1.5 \mathrm{TeV}$. We prefer to a small fine-tuning measure $\Delta_{F T}$, which can be realized in B-LSSM. Therefore, the physical Higgs boson mass, which is mainly affected by stop quark masses, will be more naturally obtained in the B-LSSM.

The updated experimental data from run II searches at $13 \mathrm{TeV}$ with 139/fb statistics indicates the mass of $Z^{\prime}$ boson satisfies $M_{Z}^{\prime} \geq 5.1 \mathrm{TeV}$ with $95 \%$ confidence level(CL) [53]. Refs. [54, 55] give us an upper bound on the ratio between the mass of $Z^{\prime}$ boson and its gauge coupling at $99 \% \mathrm{CL}$ as $\frac{M_{Z}^{\prime}}{g_{B}} \geq 6 \mathrm{TeV}$. We choose $M_{Z}^{\prime}=5.1 \mathrm{TeV}$ in our numerical calculation, so the value of parameter $g_{B}$ is restricted in the region of $0<g_{B} \leq 0.85$. The Yukawa coupling $Y_{b}$, determined by the parameter $t b$, is defined as $Y_{b}=\sqrt{2\left(t b^{2}+1\right)} m_{b} / v$. In general, the value of $Y_{b}$ is smaller than $1, m_{b} \simeq 4.18 \mathrm{GeV}$ and $v \simeq 246 \mathrm{GeV}$, so the parameter $t b$ should be approximatively smaller than 40 . Besides, the large $t b$ has been excluded by the $\bar{B} \rightarrow X_{s} \gamma$ experiment $[56,57]$. The coupling parameter $g_{Y B}$ will be taken around $-0.45 \sim-0.05$, and the reason why constant $g_{Y B}$ is negative has been discussed specifically in appendix C [58]. In addition, LHC searches constrain $t b^{\prime}<1.5[58,59]$.

We reanalyze the influence of free parameters on Higgs mass. Let the free parameters be randomly valued within the reasonable intervals: $t b \in(5,40), t b^{\prime} \in(1.02,1.5), g_{B} \in$ $(0.1,0.9), g_{Y B} \in(-0.8,0.05), B_{\eta} \in(0.405,1.6) \mathrm{TeV}^{2}, m_{\tilde{t}}, m_{\tilde{b}} \in(1.6,4) \mathrm{TeV}$ and $\mu_{\eta}, A_{t}, A_{b} \in$ $(0.2,2) \mathrm{TeV}$, we analyze the effect of parameters on the mass of Higgs boson. We consider the following constraints on the numerical results: $B \rightarrow X_{s} \gamma,(g-2)_{\mu}$ and Higgs decay ratio all satisfy the experimental deviation of $2 \sigma$, physical Higgs boson mass satisfies the experimental deviation of $3 \sigma$, and the second light Higgs boson mass is greater than $350 \mathrm{GeV}$. At this time, we find some sensitive free parameters. As shown in figure 1, we first analyze the changes of parameters $t b$ versus $t b^{\prime}$ and $g_{B}$ versus $g_{Y B}$. It is not difficult to find that when the corresponding experimental limitations are met, $14<t b<40$ and $1.3<t b^{\prime}<1.5$. Besides, the range of $g_{Y B}$ is not limited by the constraints considered above when $g_{B}>0.6$. When $g_{Y B}$ approaches to bigger than -0.4, the allowed range of $g_{B}$ reads $0.1<g_{B}<0.8$.

Then, we analyze the influence on the mass of the second light Higgs boson versus the parameters $t b^{\prime}$ and $B_{\eta}$ respectively in figure 2. Parameters $t b^{\prime}$ and $B_{\eta}$ both affect the second light Higgs boson mass smartly. The larger $t b^{\prime}$, the larger mass of the second light Higgs boson we can obtain. Parameter $B_{\eta}$ possesses the same character as parameter $t b^{\prime}$. 

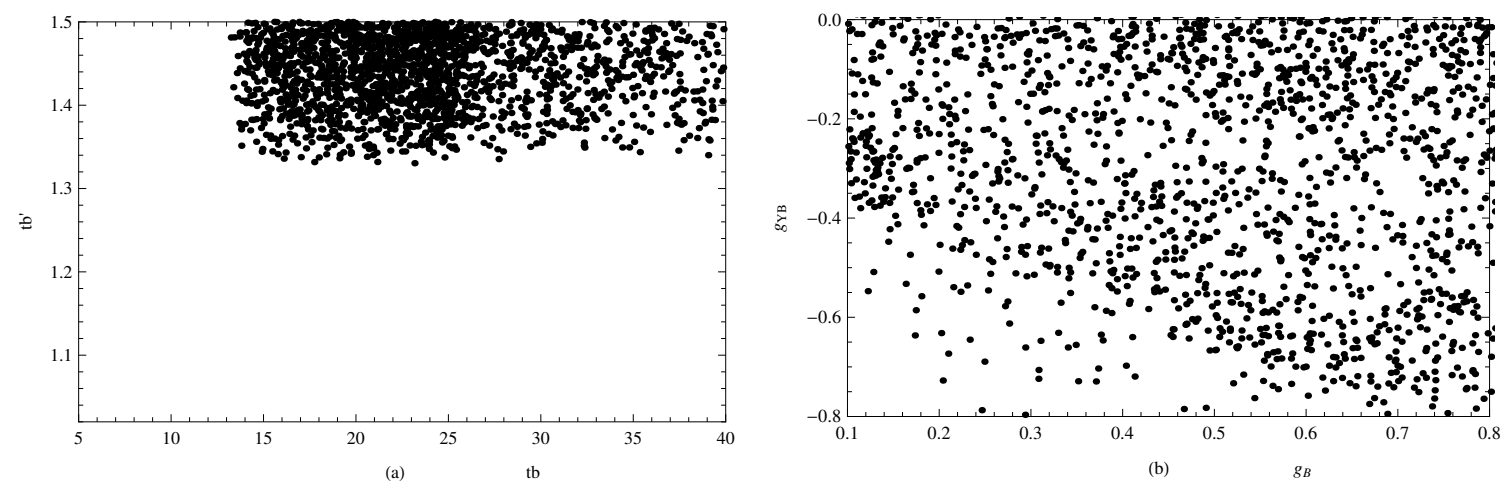

Figure 1. The changes of parameters $t b$ versus $t b^{\prime}$ and $g_{B}$ versus $g_{Y B}$.
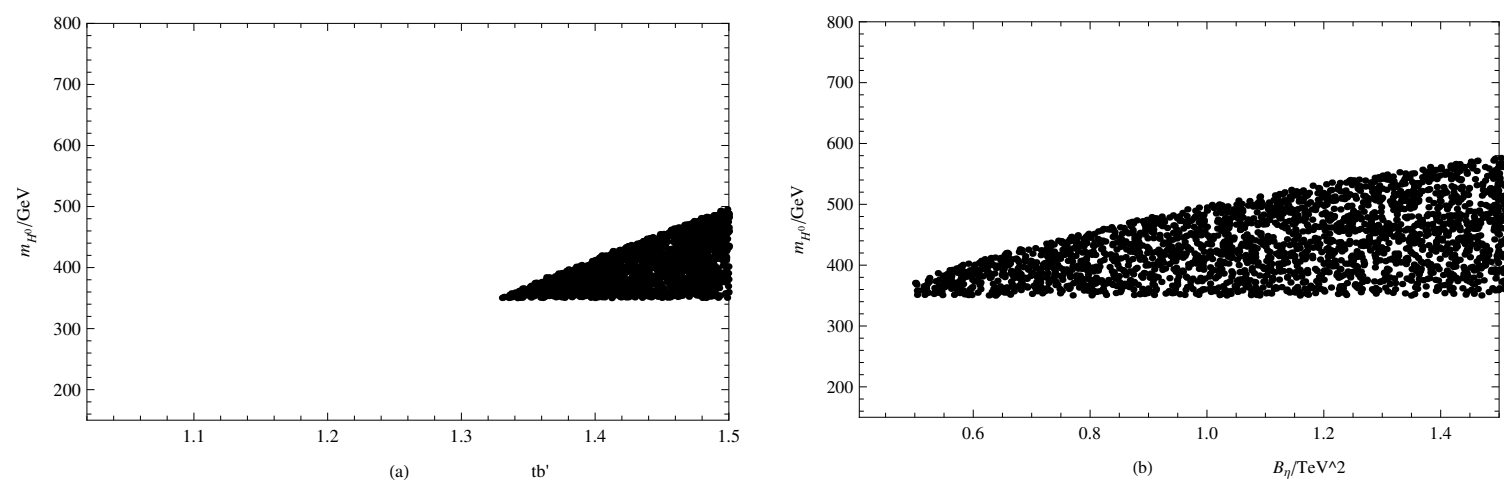

Figure 2. The second light Higgs boson masses change with the parameters $t b^{\prime}$ and $B_{\eta}$ respectively.

\section{Finite temperature effective potential in the B-LSSM}

In the B-LSSM, in the basis $\left(B_{\mu}, W_{\mu}^{3}, B_{\mu}^{\prime \prime}\right)$, we can obtain the corresponding gauge boson mass matrix. Then we deduce the exact eigenvalues of $Z^{\prime}$ boson: $m_{Z^{\prime}}^{2}=\frac{1}{8}\left(\left(g_{1}^{2}+g_{2}^{2}+\right.\right.$ $\left.\left.g_{Y B}^{2}\right) v^{2}+4 g_{B}^{2} u^{2}+\sqrt{\left(g_{1}^{2}+g_{2}^{2}+g_{Y B}^{2}\right)^{2} v^{4}+8\left(g_{Y B}^{2}-g_{1}^{2}-g_{2}^{2}\right) g_{B}^{2} v^{2} u^{2}+16 g_{B}^{4} u^{4}}\right)$. The mass of $Z^{\prime}$ boson will be reduced as $m_{Z^{\prime}}^{2} \simeq \frac{1}{4} g_{Y B}^{2} v^{2}+g_{B}^{2} u^{2} . v \simeq 246 \mathrm{GeV},-0.45<g_{Y B}<-0.05$, $m_{Z^{\prime}}>5.1 \mathrm{TeV}$ is restricted by the run II searches at $13 \mathrm{TeV}$ with $139 / \mathrm{fb}$ statistics and $0<g_{B}<0.85$ is limited through refs. $[54,55]$. The value of $\mathrm{U}(1)_{B-L}$ breaking Higgs singlets VEV $u$ is $u \simeq m_{Z^{\prime}} / g_{B} \simeq 10 \mathrm{TeV}$, which is much larger than the Higgs doublets VEV $v \simeq 246 \mathrm{GeV}$. When the temperature of the universe is higher than the scale of the new physics, both $\mathrm{U}(1)_{B-L}$ Higgs singlets and Higgs doublets are supposed to be trapped at the origins of their potential. As the temperature drops down to the scale of the new physics, the first-order PT with the two Higgs singlets may occur because the scale of Higgs potential becomes the new physics one. The scale of new physics is much higher than the electroweak scale, so we ignore two Higgs doublets part and their interaction terms in the following theoretical analyses and numerical discussions. Then, we consider the tree-level 
scalar potential including the two singlet superfields $\hat{\eta}$ and $\hat{\bar{\eta}}$, which will be written as

$$
\begin{aligned}
V_{0}= & \frac{1}{2}\left[g_{B}\left(\frac{1}{6} Q^{I *} Q^{I}-\frac{1}{6} D^{I *} D^{I}-\frac{1}{6} U^{I *} U^{I}-\frac{1}{2} L^{I *} L^{I}+\frac{1}{2} E^{I *} E^{I}+\frac{1}{2} \nu^{I *} \nu^{I}-\eta^{\dagger} \eta+\bar{\eta}^{\dagger} \bar{\eta}\right)\right. \\
& \left.+\frac{1}{2} g_{Y B}\left(\frac{1}{3} Q^{I *} Q^{I}+\frac{2}{3} D^{I *} D^{I}-\frac{4}{3} U^{I *} U^{I}-L^{I *} L^{I}+2 E^{I *} E^{I}\right)\right]^{2}+\left|\mu_{\eta}\right|^{2} \eta^{\dagger} \eta+\left|\mu_{\eta}\right|^{2} \bar{\eta}^{\dagger} \bar{\eta} \\
& -\left(\mu_{\eta} Y_{x} \bar{\eta}^{\dagger} \nu^{I} \nu^{I}+\text { H.c. }\right)+Y_{x}^{2}\left|\nu^{I} \nu^{I}\right|^{2}+4 Y_{x}^{2}\left|\eta \nu^{I}\right|^{2}+m_{\eta}^{2} \eta^{\dagger} \eta+m_{\bar{\eta}}^{2} \bar{\eta}^{\dagger} \bar{\eta}-\left(B_{\eta} \eta \bar{\eta}+\text { H.c. }\right) .
\end{aligned}
$$

Here, the contribution of $-B_{\eta} \eta \bar{\eta}$ is deduced from the softbreaking terms, and parameter $B_{\eta}$ possesses mass square dimension.

\subsection{Finite temperature effective potential}

The GW spectrum is generated by the first-order PT, which is determined by the finite temperature effective potential. In the B-LSSM, the finite temperature effective potential for both zero and finite temperatures are essential for realizing the first-order PT. We are actually interested in the $\operatorname{Re}(\eta) \equiv \frac{1}{\sqrt{2}} \phi_{\eta}$ and $\operatorname{Re}(\bar{\eta}) \equiv \frac{1}{\sqrt{2}} \phi_{\bar{\eta}}$. Therefore, the finite temperature effective potential with the CP-even Higgs components $\phi_{\eta}$ and $\phi_{\bar{\eta}}$ can be expressed as

$$
V_{\text {eff }}\left(\phi_{\eta}, \phi_{\bar{\eta}}, T\right)=V_{0}\left(\phi_{\eta}, \phi_{\bar{\eta}}\right)+\Delta V_{1}\left(\phi_{\eta}, \phi_{\bar{\eta}}, 0\right)+\Delta V_{1}\left(\phi_{\eta}, \phi_{\bar{\eta}}, T\right)+\Delta V_{\text {daisy }}\left(\phi_{\eta}, \phi_{\bar{\eta}}, T\right),
$$

where $V_{0}\left(\phi_{\eta}, \phi_{\bar{\eta}}\right)$ and $\Delta V_{1}\left(\phi_{\eta}, \phi_{\bar{\eta}}, 0\right)$ represent the tree-level and one-loop zero temperature effective potential respectively. $\Delta V_{1}\left(\phi_{\eta}, \phi_{\bar{\eta}}, T\right)$ are the one-loop finite temperature contributions, and $\Delta V_{\text {daisy }}\left(\phi_{\eta}, \phi_{\bar{\eta}}, T\right)$ are the daisy corrections. The concrete expressions will be concluded as $[60,61]$

$$
\begin{gathered}
V_{0}\left(\phi_{\eta}, \phi_{\bar{\eta}}\right)=\frac{1}{2}\left[\frac{1}{4} g_{B}^{2}\left(\phi_{\eta}^{*} \phi_{\eta}-\phi_{\bar{\eta}}^{*} \phi_{\bar{\eta}}\right)^{2}+\left(\left|\mu_{\eta}\right|^{2}+m_{\eta}^{2}\right) \phi_{\eta}^{*} \phi_{\eta}\right. \\
\left.+\left(\left|\mu_{\eta}\right|^{2}+m_{\bar{\eta}}^{2}\right) \phi_{\bar{\eta}}^{*} \phi_{\bar{\eta}}+\left(-B_{\eta} \phi_{\eta} \phi_{\bar{\eta}}+\text { H.c. }\right)\right], \\
\Delta V_{1}\left(\phi_{\eta}, \phi_{\bar{\eta}}, 0\right)=\sum_{i}(-1)^{k} \frac{n_{i}}{64 \pi^{2}} m_{i}^{4}\left(\phi_{\eta}, \phi_{\bar{\eta}}\right)\left[\log \frac{m_{i}^{2}\left(\phi_{\eta}, \phi_{\bar{\eta}}\right)}{\Lambda^{2}}-c_{i}\right], \\
\Delta V_{1}\left(\phi_{\eta}, \phi_{\bar{\eta}}, T\right)=\frac{T^{4}}{2 \pi^{2}} \sum_{i}(-1)^{k} n_{i} J_{i}\left[\frac{m_{i}^{2}\left(\phi_{\eta}, \phi_{\bar{\eta}}\right)}{T^{2}}\right], \\
\Delta V_{\text {daisy }}\left(\phi_{\eta}, \phi_{\bar{\eta}}, T\right)=-\frac{T}{12 \pi} \sum_{i=\text { boson }} n_{i}\left[\mathcal{M}_{i}^{3}\left(\phi_{\eta}, \phi_{\bar{\eta}}, T\right)-m_{i}^{3}\left(\phi_{\eta}, \phi_{\bar{\eta}}\right)\right] .
\end{gathered}
$$

$m_{i}^{2}$ are the particle masses square of fermions and bosons with the CP-even Higgs components $\phi_{\eta}$ and $\phi_{\bar{\eta}} . n_{i}$ are the degrees of freedom corresponding to the particles. $k=1(0)$ and $c_{i}=\frac{3}{2}\left(\frac{5}{6}\right)$ correspond to fermions (bosons). The thermal function corresponding to boson and fermion particles can be written as $J_{\text {boson }}\left(x^{2}\right)=\int_{0}^{\infty} d y y^{2} \log \left(1-\exp \left[y^{2}+x^{2}\right]\right)$ and $J_{\text {fermion }}\left(x^{2}\right)=\int_{0}^{\infty} d y y^{2} \log \left(1+\exp \left[y^{2}+x^{2}\right]\right)$. The masses square $\mathcal{M}_{i}^{2}$ are obtained from the $m_{i}^{2}$ by adding the T-dependent self-energy corrections [62-65]. We will discuss the $\left(\phi_{\eta}, \phi_{\bar{\eta}}\right)$-dependent particle masses square $m_{i}^{2}$ and $\mathcal{M}_{i}^{2}$ specifically as follows. 
The $\left(\phi_{\eta}, \phi_{\bar{\eta}}\right)$-dependent masses square of the CP-even Higgs and CP-odd Higgs are shown as

$$
\begin{array}{ll}
m_{\phi_{\eta} \phi_{\eta}}^{2}=g_{B}^{2} \phi_{\eta}^{2}+B_{\eta} \frac{\phi_{\bar{\eta}}}{\phi_{\eta}}, & m_{\phi_{\bar{\eta}} \phi_{\bar{\eta}}}^{2}=g_{B}^{2} \phi_{\bar{\eta}}^{2}+B_{\eta} \frac{\phi_{\eta}}{\phi_{\bar{\eta}}} \\
m_{\sigma_{\eta} \sigma_{\eta}}^{2}=B_{\eta} \frac{\phi_{\bar{\eta}}}{\phi_{\eta}}, & m_{\sigma_{\bar{\eta}} \sigma_{\bar{\eta}}}^{2}=B_{\eta} \frac{\phi_{\eta}}{\phi_{\bar{\eta}}}
\end{array}
$$

The $\left(\phi_{\eta}, \phi_{\bar{\eta}}\right)$-dependent masses square of the squarks and sleptons, CP-even and CP-odd sneutrinos can be written as

$$
\begin{aligned}
m_{\tilde{Q}^{I}}^{2} & =-\frac{1}{12}\left(g_{B}^{2}+g_{B} g_{Y B}\right)\left(\phi_{\eta}^{2}-\phi_{\bar{\eta}}^{2}\right), & & m_{\tilde{U}^{I}}^{2}=\frac{1}{12}\left(g_{B}^{2}+4 g_{B} g_{Y B}\right)\left(\phi_{\eta}^{2}-\phi_{\bar{\eta}}^{2}\right), \\
m_{\tilde{D}^{I}}^{2} & =\frac{1}{12}\left(g_{B}^{2}-2 g_{B} g_{Y B}\right)\left(\phi_{\eta}^{2}-\phi_{\bar{\eta}}^{2}\right) ; & & \\
m_{\tilde{L}^{I}}^{2} & =\frac{1}{4}\left(g_{B}^{2}+g_{B} g_{Y B}\right)\left(\phi_{\eta}^{2}-\phi_{\bar{\eta}}^{2}\right), & & m_{\tilde{R}^{I}}^{2}=-\frac{1}{4}\left(g_{B}^{2}+2 g_{B} g_{Y B}\right)\left(\phi_{\eta}^{2}-\phi_{\bar{\eta}}^{2}\right), \\
m_{\tilde{\nu}^{i}}^{2} & =m_{\tilde{\nu}_{i}^{R}}^{2}=-\frac{1}{4} g_{B}^{2}\left(\phi_{\eta}^{2}-\phi_{\bar{\eta}}^{2}\right) . & &
\end{aligned}
$$

In the B-LSSM, there is a $\left(\phi_{\eta}, \phi_{\bar{\eta}}\right)$-dependent $\mathrm{U}(1)_{B-L}$ gauge boson, whose mass square is given by

$$
m_{Z^{\prime}}^{2}=g_{B}^{2}\left(\phi_{\eta}^{2}+\phi_{\bar{\eta}}^{2}\right)
$$

In the basis $\left(\tilde{B}^{\prime}, \tilde{\eta}, \tilde{\bar{\eta}}\right)$, we obtain the neutralino mass matrix, which is a Majorana fermionic component.

$$
m_{\tilde{\chi}^{0}}=\left(\begin{array}{ccc}
0 & -g_{B} \phi_{\eta} & g_{B} \phi_{\bar{\eta}} \\
-g_{B} \phi_{\eta} & 0 & 0 \\
g_{B} \phi_{\bar{\eta}} & 0 & 0
\end{array}\right)
$$

Diagonalizing the $m_{\tilde{\chi}^{0}}^{\dagger} m_{\tilde{\chi}^{0}}$, we can obtain the corresponding mass eigenvalues, which are $g_{B}^{2}\left(\phi_{\eta}^{2}+\phi_{\bar{\eta}}^{2}\right), g_{B}^{2}\left(\phi_{\eta}^{2}+\phi_{\bar{\eta}}^{2}\right)$ and 0.

Then, considering the $T^{2}$-proportional part of the one-loop corrections, we adopt the mass square $\mathcal{M}_{i}^{2}$ for particles such as the Higgs boson, squark, slepton, sneutrino and $Z^{\prime}$ boson. Here, the temperature corrections for the third squarks consider the Yukawa couplings $Y_{t}$ and $Y_{b}$, which are both determined by the parameter $t b$. Besides, we only 
consider the temperature contributions to the longitudinal component of $Z^{\prime}$ boson.

$$
\begin{aligned}
\mathcal{M}_{\phi_{\eta} \phi_{\eta}}^{2} & =m_{\phi_{\eta} \phi_{\eta}}^{2}+\frac{T^{2}}{2} g_{B}^{2}, \quad \mathcal{M}_{\phi_{\bar{\eta}} \phi_{\bar{\eta}}}^{2}=m_{\phi_{\bar{\eta}} \phi_{\bar{\eta}}}^{2}+\frac{T^{2}}{2} g_{B}^{2} \\
\mathcal{M}_{\sigma_{\eta} \sigma_{\eta}}^{2} & =m_{\sigma_{\eta} \sigma_{\eta}}^{2}+\frac{T^{2}}{2} g_{B}^{2}, \quad \mathcal{M}_{\sigma_{\bar{\eta}} \sigma_{\bar{\eta}}}^{2}=m_{\sigma_{\bar{\eta}} \sigma_{\bar{\eta}}}^{2}+\frac{T^{2}}{2} g_{B}^{2} \\
\mathcal{M}_{\tilde{Q}^{1,2}}^{2} & =m_{\tilde{Q}^{1,2}}^{2}+\frac{T^{2}}{8}\left(\frac{16}{3} g_{s}^{2}+3 g_{2}^{2}+\frac{1}{3} g_{1}^{2}+\frac{1}{36}\left(4 g_{B}^{2}+4 g_{Y B}^{2}+7 g_{B} g_{Y B}\right)\right) \\
\mathcal{M}_{\tilde{Q}^{3}}^{2} & =m_{\tilde{Q}^{3}}^{2}+\frac{T^{2}}{8}\left(\frac{16}{3} g_{s}^{2}+3 g_{2}^{2}+\frac{1}{3} g_{1}^{2}+2\left(Y_{t}^{2}+Y_{b}^{2}\right)+\frac{1}{36}\left(4 g_{B}^{2}+4 g_{Y B}^{2}+7 g_{B} g_{Y B}\right)\right) \\
\mathcal{M}_{\tilde{U}^{1,2}}^{2} & =m_{\tilde{U}^{1,2}}^{2}+\frac{T^{2}}{8}\left(\frac{16}{3} g_{s}^{2}+\frac{16}{9} g_{1}^{2}+\frac{1}{9}\left(g_{B}^{2}+16 g_{Y B}^{2}+7 g_{B} g_{Y B}\right)\right) \\
\mathcal{M}_{\tilde{U}^{3}}^{2} & =m_{\tilde{U}^{3}}^{2}+\frac{T^{2}}{8}\left(\frac{16}{3} g_{s}^{2}+\frac{16}{9} g_{1}^{2}+4 Y_{t}^{2}+\frac{1}{9}\left(g_{B}^{2}+16 g_{Y B}^{2}+7 g_{B} g_{Y B}\right)\right) \\
\mathcal{M}_{\tilde{D}^{1,2}}^{2} & =m_{\tilde{D}^{1,2}}^{2}+\frac{T^{2}}{8}\left(\frac{16}{3} g_{s}^{2}+\frac{1}{3} g_{1}^{2}+\frac{1}{9}\left(g_{B}^{2}+4 g_{Y B}^{2}+\frac{5}{2} g_{B} g_{Y B}\right)\right) \\
\mathcal{M}_{\tilde{D}^{3}}^{2} & =m_{\tilde{D}^{3}}^{2}=+\frac{T^{2}}{8}\left(\frac{16}{3} g_{s}^{2}+\frac{1}{3} g_{1}^{2}+4 Y_{b}^{2}+\frac{1}{9}\left(g_{B}^{2}+4 g_{Y B}^{2}+\frac{5}{2} g_{B} g_{Y B}\right)\right) \\
\mathcal{M}_{\tilde{L}^{I}}^{2} & =m_{\tilde{L}^{I}}^{2}+\frac{T^{2}}{8}\left(3 g_{2}^{2}+g_{1}^{2}+\frac{1}{4}\left(4 g_{B}^{2}+4 g_{Y B}^{2}+7 g_{B} g_{Y B}\right)\right) \\
\mathcal{M}_{\tilde{R}^{I}}^{2} & =m_{\tilde{R}^{I}}^{2}+\frac{T^{2}}{8}\left(4 g_{1}^{2}+\frac{1}{4}\left(4 g_{B}^{2}+7 g_{Y B}^{2}+14 g_{B} g_{Y B}\right)\right) \\
\mathcal{M}_{\tilde{\nu}_{i}}^{2} & =\mathcal{M}_{\tilde{\nu}_{i}^{R}}^{2}=m_{\tilde{\nu}_{i}}^{2}+\frac{T^{2}}{8} g_{B}^{2} \\
\mathcal{M}_{Z^{\prime}}^{2} & =m_{Z^{\prime}}^{2}+\frac{T^{2}}{2}\left(2 g_{B}^{2}+g_{Y B}^{2}\right)
\end{aligned}
$$

\subsection{The PT from the finite temperature effective potential with the CP-even Higgs components $\phi_{\eta}$ and $\phi_{\bar{\eta}}$}

We analyze the PT from the finite temperature effective potential with the CP-even Higgs components $\phi_{\eta}$ and $\phi_{\bar{\eta}}$. In order to discuss the PT easier, we make the approximate assumption as follows: $\frac{\phi_{\bar{\eta}}}{\phi_{\eta}}=t b^{\prime}$ and $\phi=\sqrt{\phi_{\bar{\eta}}^{2}+\phi_{\eta}^{2}}$. Then the finite temperature effective potential $V_{\text {eff }}\left(\phi_{\eta}, \phi_{\bar{\eta}}, T\right)$ will be deduced as $V_{\text {eff }}(\phi, T)$.

With the parameters $t b^{\prime}=1.35, g_{B}=0.5, g_{Y B}=-0.2, \mu_{\eta}=600 \mathrm{GeV}, B_{\eta}=1.3 \mathrm{TeV}^{2}$, $m_{\eta}=1.3 \mathrm{TeV}$ and $m_{\bar{\eta}}=300 \mathrm{GeV}$, we plot the finite temperature effective potential $V_{\text {eff }}(\phi, T)$ changing with $\phi$ in figure 3 at critical temperature $T_{c} \simeq 1.65 \mathrm{TeV}$. We will easily find that the effective potential $V_{\text {eff }}(\phi, T)$ possesses the minimum both at $\phi=0 \mathrm{GeV}$ and $\phi=v_{c} \simeq 1.75 \mathrm{TeV}$ when $T_{c} \simeq 1.65 \mathrm{TeV}$. Besides, $v_{c} / T_{c}>1$, which can satisfy the strong first-order PT. The critical temperature is around $1.65 \mathrm{TeV}$, which is much larger than the electroweak scale, so we mainly discuss the GW from the strong first-order PT with the CP-even Higgs components $\phi_{\eta}$ and $\phi_{\bar{\eta}}$. With the temperature continue decreasing and around the electroweak scale, the electroweak symmetry breaks down. 


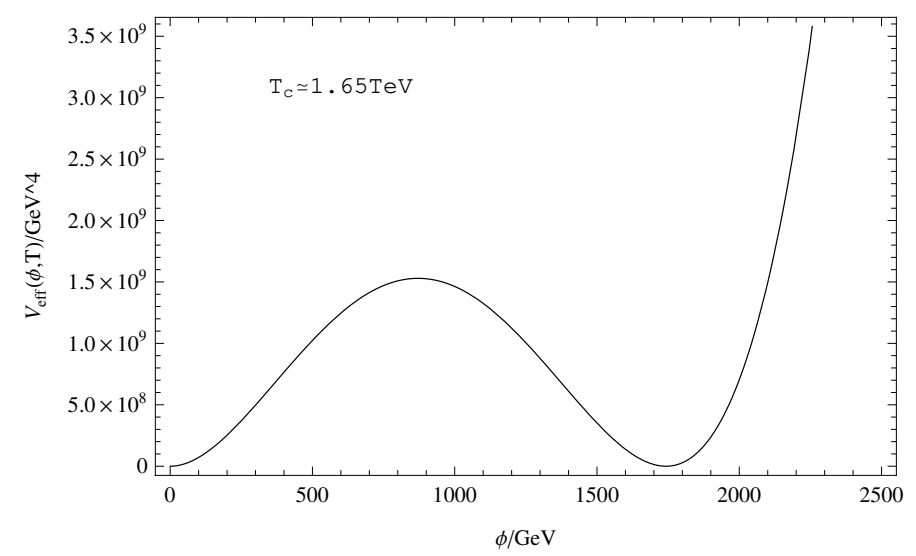

Figure 3. The finite temperature effective potential $V_{\text {eff }}(\phi, T)$ changes with $\phi$ at $T_{c} \simeq 1.65 \mathrm{TeV}$.

\section{GW generation by cosmological first-order PT}

The breaking of B-L symmetry not only induces first-order PT, but also induces secondorder PT. The first-order PT is from a false vacuum state(symmetry phase) to a true one(symmetry broken phase), while the second-order PT is the process from an unstable state to a vacuum state.

The baryon asymmetry of the universe can be generated via the baryogenesis mechanism, which requires a strong first-order PT to provide a system deviation from thermal equilibrium. First-order PT will generate bubbles, inside which are symmetry broken phases, their externals are the symmetry phases. The universe begins with the symmetry phase, which is a metastable one. With the expansion of the bubbles, the collision and then fusion, the universe will reside at the symmetry broken phase. The GW will be generated along with the collision between bubbles, the plasma movement near the bubble wall disturbed by the expanding bubbles, and the magnetofluid turbulence caused by the plasma. The relative height of potential between false vacuum state and true one will determine the strength of GW. The GW can be detectable with the large enough relative height of potential, and the corresponding PT is called as the strong first-order PT.

The second-order PT can also generate bubbles initially. However, the background is unstable and will quickly roll to the vacuum state, so the bubbles and background will be exactly the same soon. The second-order PT will be more synchronous than the first-order one. Therefore, there are almost no bubble collision, plasma turbulence even the GW in the second-order PT. Therefore, we pay attention to the strong first-order PT to study the GW signals within the B-LSSM.

\subsection{Scalar potential parameters related to the GW spectrum}

In this section, we briefly discuss the properties of the GWs, which critically depend on two quantities: the strength parameter $\alpha_{\theta}$, which will be calculated using the trace of energy momentum tensor; $\beta$ is the speed of the $\mathrm{PT}$ at the nucleation temperature $T_{n}$. The transition from the symmetric phase to the symmetric broken phase takes place via thermal 
tunneling at the finite temperature. First-order PT pushes the bubbles of the symmetric broken phase to be nucleated, then they expand and eventually fill up the entire universe. The bubble nucleation rate per unit volume at the finite temperature is given by

$$
\Gamma \sim A(T) e^{-S}=A(T) e^{-S_{E} / T} .
$$

$A(T)$ is a factor that is roughly proportional to $T^{4}$. $S$ represents the action in the fourdimensional Minkowski space, while $S_{E}$ is the three-dimensional Euclidean action [66, 67]. Parameter $\beta$ can be defined as:

$$
\beta \equiv-\left.\left.\frac{d S}{d t}\right|_{t=t_{n}} \simeq H_{n} T \frac{d S}{d T}\right|_{T=T_{n}}=\left.H_{n} T \frac{d\left(S_{E} / T\right)}{d T}\right|_{T=T_{n}},
$$

where $H_{n}$ denotes the Hubble rate at the nucleation temperature $T_{n}$. The key parameter that controls the GW signals is $\beta / H_{n}$. And the smaller $\beta / H_{n}$ will lead to the stronger PT and consequently the more sensitive GW signals.

Then, referencing [68] and [69], we use the trace of the energy momentum tensor to calculate the strength parameter $\alpha_{\theta}$. The hydrodynamics in the plasma will be described by the energy density $\mathcal{E}$, the pressure density $p$ and the enthalpy $\omega$. The pressure $p$ is determined by the free energy $\mathcal{F}$, which is the finite temperature effective potential $V_{\text {eff }}$ : $p=-\mathcal{F}=-V_{\text {eff }}$. Then the energy $\mathcal{E}$ and enthalpy $\omega$ will be obtained respectively from the pressure density: $\mathcal{E}=T \frac{\partial p}{\partial T}-p$ and $\omega=T \frac{\partial p}{\partial T}=p+\mathcal{E}$.

The energy-momentum tensor of the plasma will be given by $T^{\mu \nu}=u^{\mu} u^{\nu} \omega+g^{\mu \nu} p$ with $u^{\mu}$ being the four-velocity of the fluid and $g^{\mu \nu}$ being the inverse Minkowski metric. Then the strength parameter $\alpha_{\theta}$ will be deduced using the trace of the energy momentum tensor $\left(\theta=g_{\mu \nu} T^{\mu \nu}\right)[70-73]: \alpha_{\theta}=\frac{\Delta \theta}{3 \omega_{+}\left(T_{n}\right)}$. Here, $\Delta \theta=\Delta \mathcal{E}-3 \Delta p, \Delta$ denotes the difference between symmetric and broken phase and $\omega_{+}\left(T_{n}\right)$ corresponds to the enthalpy of the symmetric phase at nucleation temperature $T_{n}$. We prefer to a larger value of $\alpha_{\theta}$, which will produce a much stronger PT, even a stronger GW spectrum.

In figure 4 , we will discuss the $\beta / H_{n}$ changing with parameters $B_{\eta}, g_{B}, m_{\eta}$ and $m_{\bar{\eta}}$ respectively. The values of $\beta / H_{n}$ decrease with the enlarging $B_{\eta}$, while increase with the enlarging $g_{B}, m_{\eta}$ and $m_{\bar{\eta}}$. The strength of $\mathrm{PT} \alpha_{\theta}$ versus the parameters $B_{\eta}, g_{B}, m_{\eta}$ and $m_{\bar{\eta}}$ will be researched in figure 5 respectively. With the enlarging $g_{B}$ and $m_{\eta}$, the values of $\alpha_{\theta}$ decrease. The values of $\alpha_{\theta}$ are around 0.14 as $m_{\bar{\eta}}$ in the region $300 \sim 400 \mathrm{GeV}$. Besides, the values of $\alpha_{\theta}$ will almost do not change when $B_{\eta}$ is in the region $(1.2 \sim 1.4) \mathrm{TeV}^{2}$. We prefer to the smaller $\beta / H_{n}$ and larger $\alpha_{\theta}$ to obtain the more suitable GW signals. So, we will take $\mu_{\eta}=400 \mathrm{GeV}, B_{\eta}=1.3 \mathrm{TeV}^{2}, g_{B}=0.5, m_{\eta}=1.6 \mathrm{TeV}$ and $m_{\bar{\eta}}=300 \mathrm{GeV}$ in the following numerical discussion.

\subsection{GW spectrum}

In this section, we will discuss the GW spectrums specifically. There are three sources that generate the GW spectrums from the first-order PT: (1) The initial collision of scalar field and relevant shocks in the plasma. The technique of 'envelope approximation' has been widely used to model GW power spectrum from bubble collisions, which can be denoted by $h^{2} \Omega_{\text {coll }}$. (2) After the bubbles merging, the fluid kinetic energy waves in the plasma go 

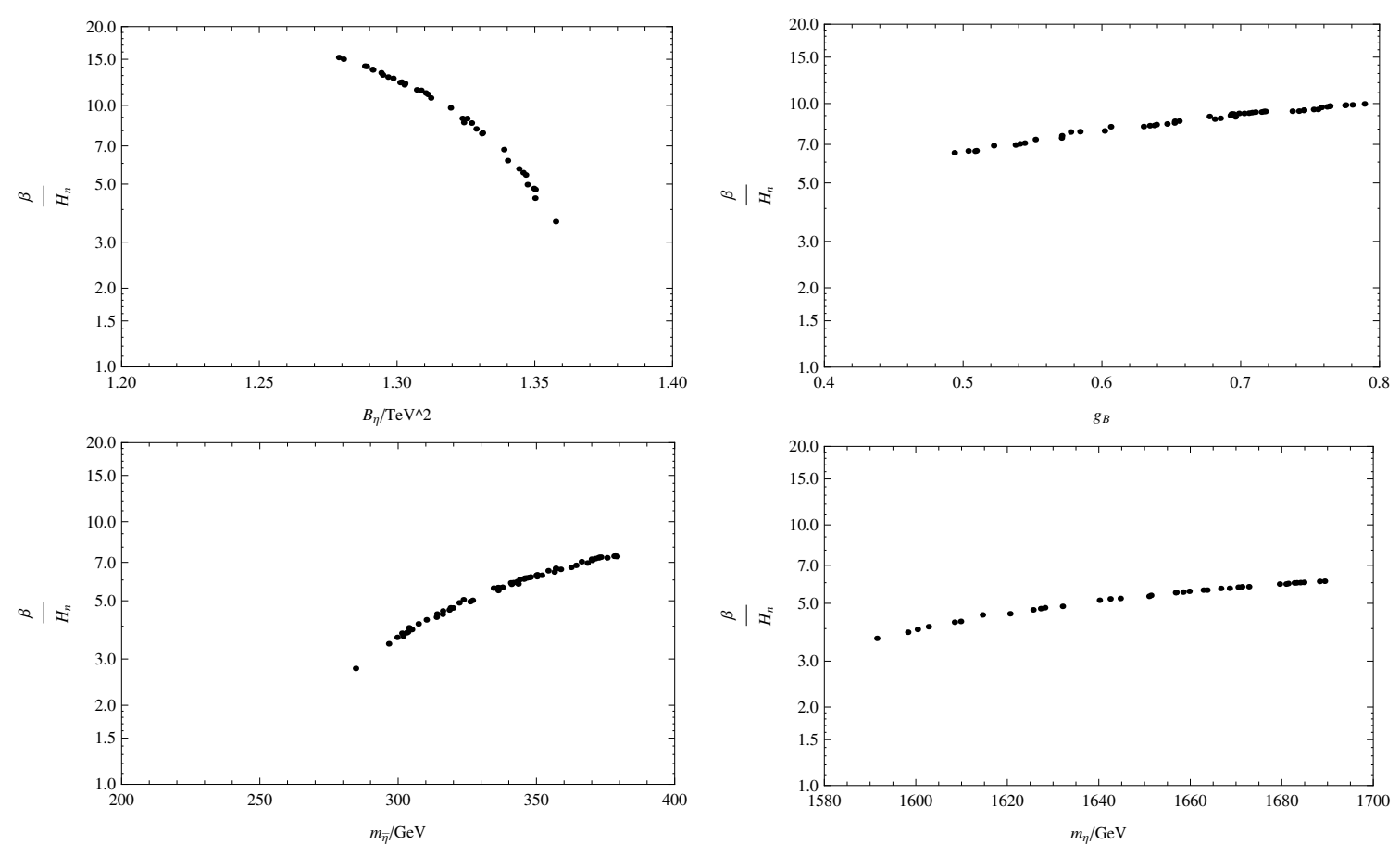

Figure 4. The values of $\beta / H_{n}$ change with the parameters $B_{\eta}, g_{B}, m_{\eta}$ and $m_{\bar{\eta}}$ respectively.
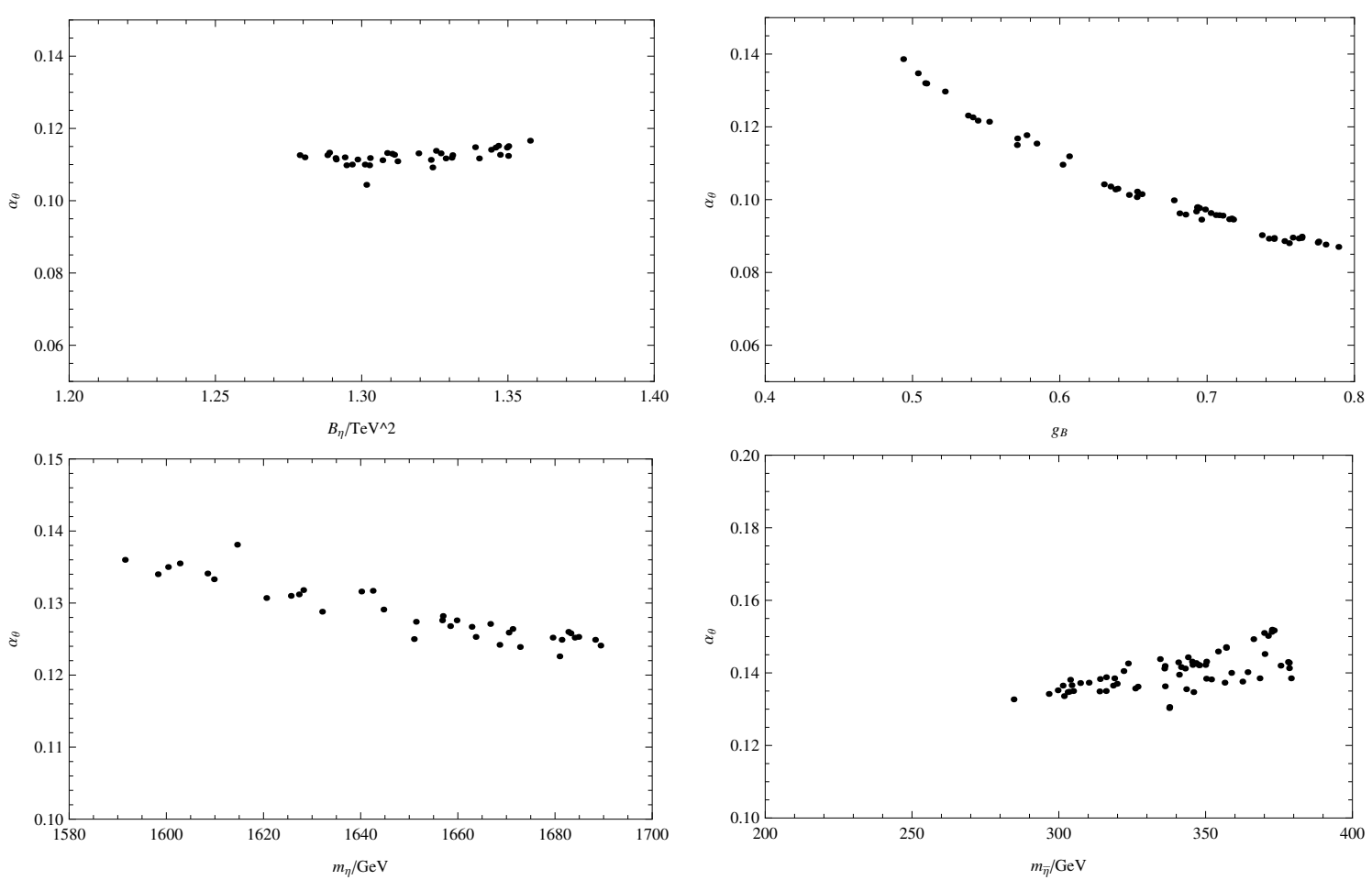

Figure 5. The strengths of PT $\alpha_{\theta}$ change with the parameters $B_{\eta}, g_{B}, m_{\eta}$ and $m_{\bar{\eta}}$ respectively. 
on propagating outward into the broken phase. These waves spread at the sound speed in the plasma without the tractive force of scalar field bubble wall. Sound waves $h^{2} \Omega_{\mathrm{sw}}$ are produced after the bubble collisions but before expansion. (3) The magnetohydrodynamic turbulent $h^{2} \Omega_{\text {trub }}$ of the surrounding plasma will be formed after the bubble collisions. These three contributions linearly combine, then we can obtain the corresponding GW spectrums, which will be expressed as

$$
h^{2} \Omega_{G W} \approx h^{2} \Omega_{\text {coll }}+h^{2} \Omega_{\mathrm{sw}}+h^{2} \Omega_{\mathrm{trub}} .
$$

\subsubsection{Bubble collisions}

Using the technique of 'envelope approximation', the contribution of the GWs generated from the bubble collisions is $[22,74]$

$$
h^{2} \Omega_{\text {coll }}(f)=1.65 \times 10^{-5} \kappa^{2} \Delta\left(\frac{\beta}{H_{n}}\right)^{-2}\left(\frac{\alpha_{\theta}}{1+\alpha_{\theta}}\right)^{2}\left(\frac{g_{*}}{100}\right)^{-1 / 3} \frac{(a+b)\left(f / f_{\text {coll }}\right)^{a}}{b+a\left(f / f_{\text {coll }}\right)^{(a+b)}},
$$

where $a=2.8$ and $b=1.0 . \kappa$ denotes the efficiency factor of the latent heat deposited into a thin shell with $A=0.715 . g_{*}$ corresponds to the relativistic degree of freedom in the thermal plasma at $T_{n}$. The concrete expression of $\kappa$ and $\Delta$ will be discussed as follows [22]

$$
\kappa=\frac{1}{1+A \alpha_{\theta}}\left(A \alpha_{\theta}+\frac{4}{27} \sqrt{\frac{3 \alpha_{\theta}}{2}}\right), \quad \Delta=\frac{0.11 v_{b}^{3}}{0.42+v_{b}^{2}},
$$

here, $v_{b}$ characterizes the bubble wall velocity. We choose $v_{b} \simeq 1.0$, since scenarios with nearly luminal wall velocities are more promising from the standpoint of observable gravitational radiation [33]. The peak frequency $f_{\text {coll }}$ will be determined by the characteristic time-scale of the PT. From simulations, the peak frequency at $T_{n}$ is approximately given by $\frac{f_{n}}{\beta}=\frac{0.62}{1.8-0.1 v_{b}+v_{b}^{2}}$, which is then red-shifted to yield the peak frequency today: $f_{\text {coll }}=1.67 \times 10^{-5}\left(\frac{f_{n}}{\beta}\right)\left(\frac{\beta}{H_{n}}\right)\left(\frac{T_{n}}{100 \mathrm{GeV}}\right)\left(\frac{g_{*}}{100}\right)^{1 / 6} \mathrm{~Hz}$.

\subsubsection{Sound waves}

As a more significant and long-lasting source of GW, sound wave is produced by expanding sound shells in the fluid kinetic energy after the bubble collisions. The concrete sound wave contribution to the GWs is given by $[71,75]$

$$
h^{2} \Omega_{\mathrm{sw}}(f)=h^{2} \Omega_{\mathrm{sw}}\left(f_{\mathrm{sw}}\right)\left(\frac{f}{f_{\mathrm{sw}}}\right)^{3}\left(\frac{7}{4+3\left(f / f_{\mathrm{sw}}\right)^{2}}\right)^{7 / 2},
$$

where the concrete peak amplitude $h^{2} \Omega_{\mathrm{sw}}\left(f_{\mathrm{sw}}\right)$ and the corresponding peak frequency $f_{\mathrm{sw}}$ from sound waves can be shown as

$$
\begin{aligned}
h^{2} \Omega_{\mathrm{sw}}\left(f_{\mathrm{sw}}\right) & =2.65 \times 10^{-6} \kappa_{v}^{2} v_{b}\left(\frac{\beta}{H_{n}}\right)^{-1}\left(\frac{\alpha_{\theta}}{1+\alpha_{\theta}}\right)^{2}\left(\frac{g_{*}}{100}\right)^{-1 / 3}, \\
f_{\mathrm{sw}} & =1.9 \times 10^{-5} \frac{1}{v_{b}}\left(\frac{\beta}{H_{n}}\right)\left(\frac{T_{n}}{100 \mathrm{GeV}}\right)\left(\frac{g_{*}}{100}\right)^{1 / 6} \mathrm{~Hz} .
\end{aligned}
$$

We take $\kappa_{v} \simeq \frac{\alpha_{\theta}}{0.73+0.083 \sqrt{\alpha_{\theta}}+\alpha_{\theta}}[70]$, which denotes the fraction of the latent heat transforming into the bulk motion of the fluid. Besides, efficiency $\kappa_{v}$ will be determined by the bubble expansion mode. 


\subsubsection{Turbulence}

Since the plasma is ionized fully, percolation can also induce magnetohydrodynamic turbulence in the plasma. Then, the GW contribution from the turbulence can be written as $[76,77]$

$$
\begin{aligned}
h^{2} \Omega_{\text {turb }}(f)= & 3.35 \times 10^{-4} v_{b}\left(\frac{\beta}{H_{n}}\right)^{-1}\left(\frac{\kappa_{\text {turb }} \alpha_{\theta}}{1+\alpha_{\theta}}\right)^{3 / 2}\left(\frac{g_{*}}{100}\right)^{-1 / 3} \\
& \times \frac{\left(f / f_{\text {turb }}\right)^{3}}{\left(1+f / f_{\text {turb }}\right)^{11 / 3}\left(1+8 \pi f / h_{n}\right)}
\end{aligned}
$$

where $h_{n}=1.65 \times 10^{-5}\left(\frac{T_{n}}{100 \mathrm{GeV}}\right)\left(\frac{g_{*}}{100}\right)^{1 / 6} \mathrm{~Hz}$ is the Hubble parameter today. We set the efficiency factor of the latent heat for turbulence to be $\kappa_{\text {turb }} \simeq 0.05 \kappa_{v}$ [24]. The peak frequency $f_{\text {turb }}$ of the GWs produced from the turbulence will be as follows

$$
f_{\text {turb }}=2.7 \times 10^{-5} \frac{1}{v_{b}}\left(\frac{\beta}{H_{n}}\right)\left(\frac{T_{n}}{100 \mathrm{GeV}}\right)\left(\frac{g_{*}}{100}\right)^{1 / 6} \mathrm{~Hz}
$$

\section{The numerical results of GW spectrum in the B-LSSM}

In our numerical calculation, we consider the constraints of parameter space related to the future experiment within B-LSSM. In section II, we study the Higgs masses with the one-loop zero temperature effective potential corrections. Parameters $g_{B}, g_{Y B}, t b$ and $t b^{\prime}$ have been limited within small regions, which will affect the numerical results of GW spectrum. As well as, we have studied Higgs decay modes, $B$ meson rare decay $\bar{B} \rightarrow X_{s} \gamma$ and the muon anomalous magnetic dipole moment in the B-LSSM [78]. The corresponding parameter constraints will be considered in our numerical discussion. In the following numerical discussion, we use the numerical package CosmoTransitions [79] for analyzing the corresponding PT. After calculating, we obtain the bounce solutions and discover the nucleation temperature $T_{n}$. The value of $S_{E} / T_{n}$ is around 140 below certain nucleation temperature $T_{n}$. Then we can obtain the important results of parameters $\alpha_{\theta}$ and $\beta$, which finally determine the GW spectrums.

In this part, we study the dependence of the GW spectrums on the parameters $t b$, $g_{Y B}, g_{B}, \mu_{\eta}, B_{\eta}, m_{\eta}$ and $m_{\bar{\eta}}$. First, the GW spectrums versus parameter $g_{B}$ are plotted in figure 6 with $t b=20, g_{Y B}=-0.15, \mu_{\eta}=600 \mathrm{GeV}, B_{\eta}=1.3 \mathrm{TeV}^{2}, m_{\eta}=1.6 \mathrm{TeV}$ and $m_{\bar{\eta}}=300 \mathrm{GeV}$. The color lines show the sensitivities of the future GW experiments, which include the LISA(N2A5M5L6), BBO, DECIGO and Ultimate-DECIGO. We can discover that the present and future GW observations could probe a broad parameter space for $g_{B}$. Besides, the GWs signal will be as large as $h^{2} \Omega_{G W} \sim 10^{-9}$, which can be detected by the LISA, BBO, DECIGO and Ultimate-DECIGO with $g_{B}$ in the region $(0.49 \sim 0.79)$. Then, the GW spectrums $h^{2} \Omega_{G W}$ versus frequency $f$ will be further researched in the right of figure 6 with $g_{B}=0.49$, which is preferred by the space-based experiments. The contributions to bubble collision, sound waves and turbulence are respectively plotted by 

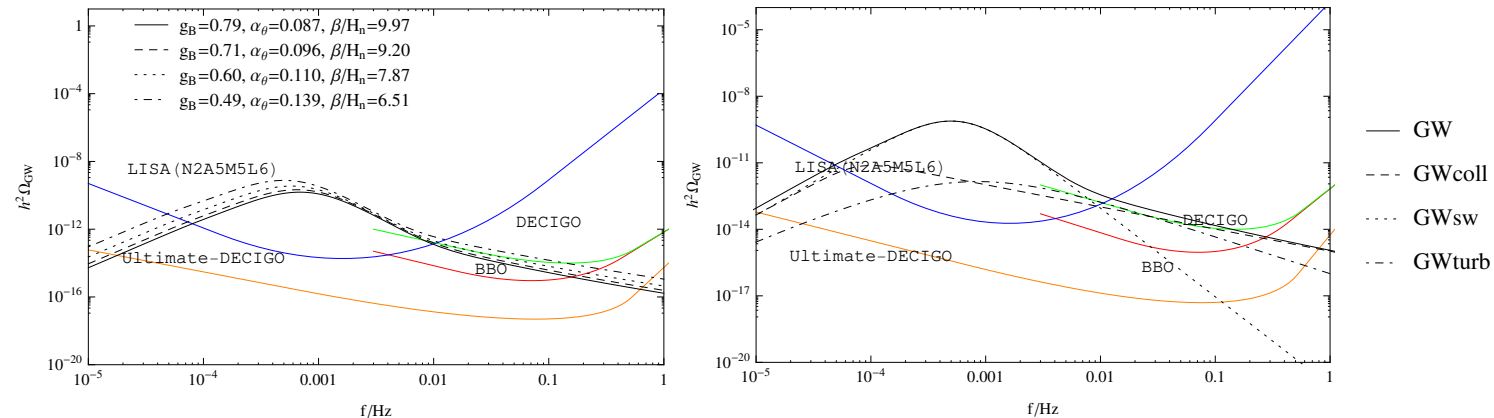

Figure 6. The contributions of GW spectrum vary with parameters $g_{B}$ in the B-LSSM.
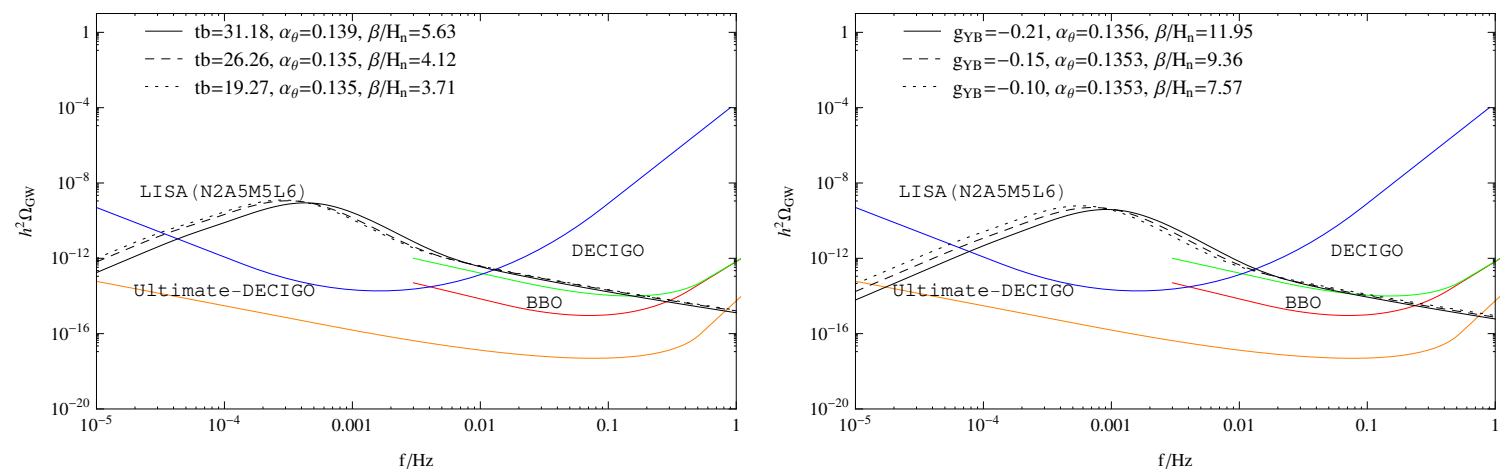

Figure 7. The contributions of GW spectrum vary with parameters $t b$ and $g_{Y B}$ in the B-LSSM.

dashed, dotted and dot dashed lines. The contributions of GW spectrum, coming from the sum of the aforementioned three contributions, will be denoted by the solid black line. We can easily find that the contribution to sound wave is dominant and almost indistinguishable from the solid black line when $f$ is around $10^{-4} \sim 0.005 \mathrm{~Hz}$.

Then, the GW spectrums versus parameters $t b$ and $g_{Y B}$ are plotted in figure 7 with $g_{B}=0.5, \mu_{\eta}=600 \mathrm{GeV}, B_{\eta}=1.3 \mathrm{TeV}^{2}, m_{\eta}=1.6 \mathrm{TeV}$ and $m_{\bar{\eta}}=300 \mathrm{GeV}$. Parameter $t b$ presents in the Yukawa couplings $Y_{t}$ and $Y_{b}$, which affects the finite temperature effective potential through the temperature corrections of the third squark masses, so that influence the GWs. We can discover that the GW spectrums can be detected by the LISA(N2A5M5L6), BBO, DECIGO and Ultimate-DECIGO as $19<t b<31$ and $-0.21<$ $g_{Y B}<-0.10$. Besides, with the decreasing $t b$ but enlarging $g_{Y B}$, the GW signal strength will increase slowly. In our following discussion, we make $t b=20$ and $g_{Y B}=-0.15$.

Parameters $m_{\eta}$ and $m_{\bar{\eta}}$ both exist in the tree-level zero temperature effective potential. When $t b=20, g_{Y B}=-0.15, g_{B}=0.5, \mu_{\eta}=600 \mathrm{GeV}$ and $B_{\eta}=1.3 \mathrm{TeV}^{2}$, the GW spectrums versus frequency $f$ with parameters $m_{\eta}$ and $m_{\bar{\eta}}$ will be shown in figure 8 . The smaller $m_{\eta}$ or $m_{\bar{\eta}}$, the larger GW spectrum with relatively smaller peak frequency we can obtain. Other than this, the value of parameter $m_{\eta}$ is around $1.6 \mathrm{TeV}$, while the value of parameter $m_{\bar{\eta}}$ is around $300 \mathrm{GeV}$. Our numerical simulations reveal that the parameters $m_{\eta}$ and $m_{\bar{\eta}}$ possess tiny parameter spaces to acquire suitable GW spectrums. 

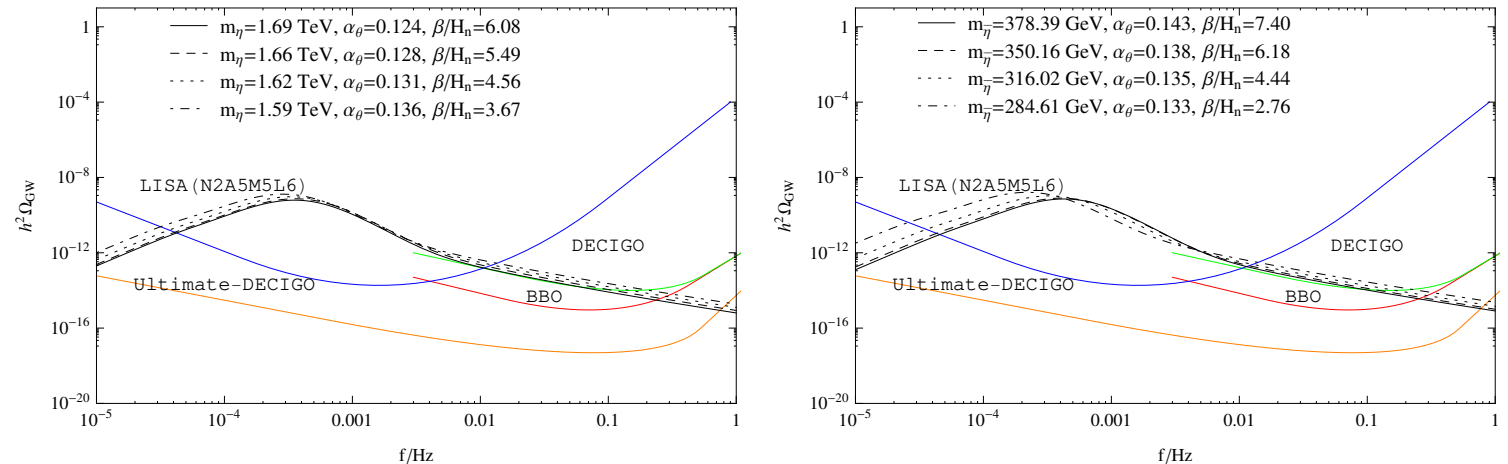

Figure 8. The contributions of GW spectrum vary with parameters $m_{\eta}$ and $m_{\bar{\eta}}$ in the B-LSSM.
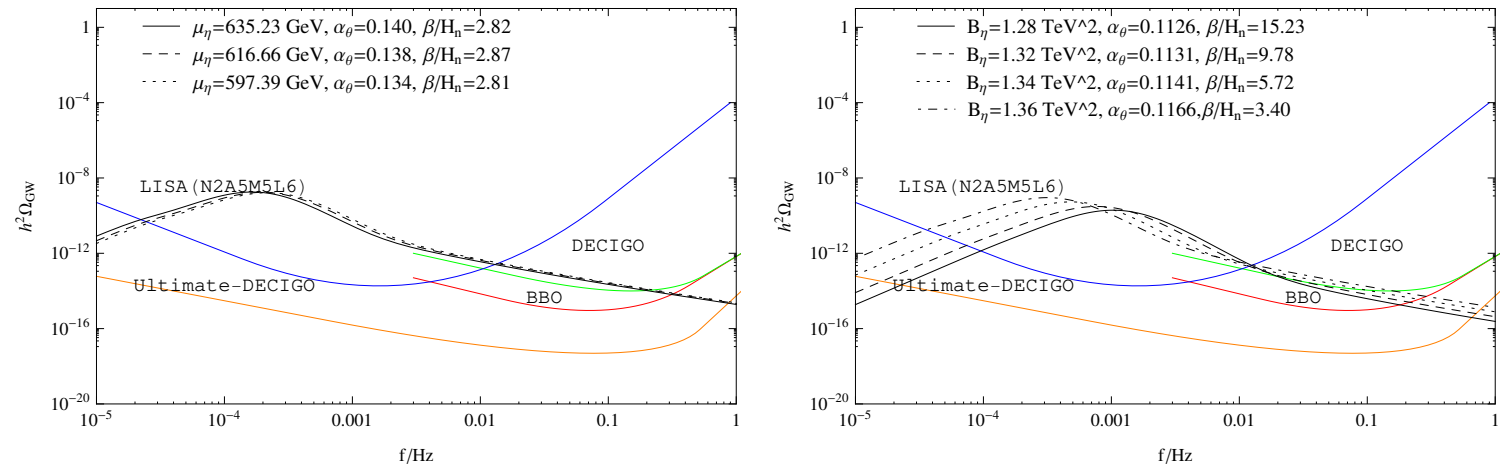

Figure 9. The contributions of GW spectrum vary with parameters $\mu_{\eta}$ and $B_{\eta}$ in the B-LSSM.

After fixing $t b=20, g_{Y B}=-0.15, g_{B}=0.5, m_{\eta}=1.6 \mathrm{TeV}$ and $m_{\bar{\eta}}=300 \mathrm{GeV}$ in the B-LSSM, the GW spectrums versus parameters $\mu_{\eta}$ and $B_{\eta}$ are demonstrated through the figure 9. As $\mu_{\eta}$ is in the region of $590 \sim 640 \mathrm{GeV}$, a larger value of $\mu_{\eta}$ tends to produce a larger GW spectrum with a relatively smaller peak frequency. It is worth noting that GW spectrum can be detected by the LISA(N2A5M5L6), BBO, DECIGO and Ultimate-DECIGO when $\mu_{\eta}$ is in the region of $590 \sim 640 \mathrm{GeV}$. Then the GW spectrum versus parameter $B_{\eta}$ will be studied. With the increasing value of $B_{\eta}$, the GW signal gradually strengthens. As $1.28 \mathrm{TeV}^{2} \leq \mathrm{B}_{\eta} \leq 1.36 \mathrm{TeV}^{2}$, GW spectrum will be detected by LISA(N2A5M5L6), BBO, DECIGO and Ultimate-DECIGO.

Through the above discussion in figure 8 and figure 9 , we find that parameters $m_{\eta}$, $m_{\bar{\eta}}, \mu_{\eta}$ and $B_{\eta}$ fluctuate within a small region. We expect to find the relationship between these parameters to explain these relatively special parameter values. So, we random scan the parameter space as $14<t b<40,-0.45<g_{Y B}<-0.01,0.1<g_{B}<0.8,200 \mathrm{GeV}<$ $\mu_{\eta}<700 \mathrm{GeV}, 0.8 \mathrm{TeV}^{2}<\mathrm{B}_{\eta}<1.6 \mathrm{TeV}^{2}, 1.2 \mathrm{TeV}<\mathrm{m}_{\eta}<2.0 \mathrm{TeV}$ and $100 \mathrm{GeV}<\mathrm{m}_{\bar{\eta}}<$ $600 \mathrm{GeV}$. When the numerical results satisfy the strong first-order PT and can obtain the suitable GW signals, we plot the ratio of random parameter $B_{\eta}$ to $\sqrt{\left(m_{\eta}^{2}+\mu_{\eta}^{2}\right)\left(m_{\bar{\eta}}^{2}+\mu_{\eta}^{2}\right)}$ in figure 10 (a). We conclude that these parameters satisfy the approximate relation: $A_{\text {tree }} \equiv$ $\frac{B_{\eta}}{\sqrt{\left(m_{\eta}^{2}+\mu_{\eta}^{2}\right)\left(m_{\bar{\eta}}^{2}+\mu_{\eta}^{2}\right)}} \simeq 1.25$. Then, we research the total contributions after considering 

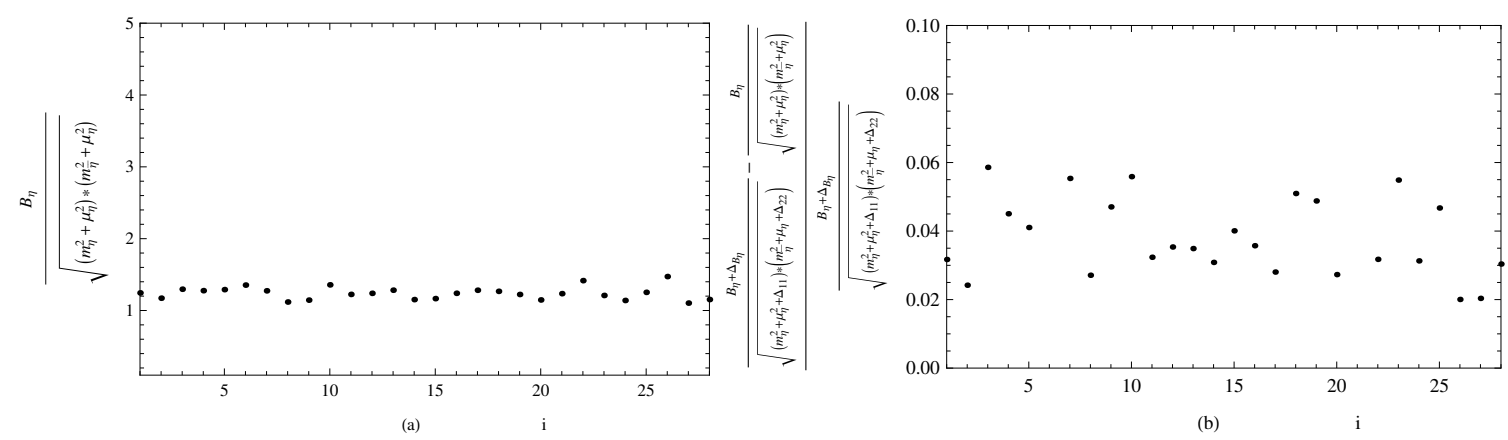

Figure 10. The tree-level and one-loop corrections of $\frac{B_{\eta}}{\sqrt{\left(m_{\eta}^{2}+\mu_{\eta}^{2}\right)\left(m_{\bar{\eta}}^{2}+\mu_{\eta}^{2}\right)}}$ versus $i$.

the one-loop correction through the one-loop zero temperature effective potential with the CP-even Higgs components $\phi_{\eta}$ and $\phi_{\bar{\eta}}: A_{\text {one-loop }} \equiv \frac{B_{\eta}+\Delta_{B_{\eta}}}{\sqrt{\left(m_{\eta}^{2}+\mu_{\eta}^{2}+\Delta_{11}\right)\left(m_{\bar{\eta}}^{2}+\mu_{\eta}^{2}+\Delta_{22}\right)}}$. The corresponding theoretical analyses will be given out in appendix E. Besides, we plot the influence of the one-loop corrections in the total contributions $\frac{A_{\text {one-loop }}-A_{\text {tree }}}{A_{\text {one-loop }}}$ in figure $10(\mathrm{~b})$, which indicates that the contributions of one-loop corrections are small and only around $2 \% \sim 6 \%$ of the total contributions.

\section{Conclusion}

In the B-LSSM, we first study the Higgs masses with the one-loop zero temperature effective potential corrections. When the physical Higgs mass satisfies $3 \sigma$ experimental interval, parameters $t b$ and $t b^{\prime}$ are limited within small regions: $14<t b<40$ and $1.3<t b^{\prime}<1.5$. In additional, the larger parameter $t b^{\prime}$ and $B_{\eta}$, the larger second-light Higgs mass we can obtain. Second, the strength parameter will be calculated using the trace of the energy momentum tensor. Then, we study the GW spectrums generated by the strong firstorder PT with the two $\mathrm{U}(1)_{B-L}$ singlet Higgs superfields. Our numerical calculations reveal that the contribution from sound wave is dominated to generate the GW signals when the frequency $f$ is around $10^{-4} \sim 0.005 \mathrm{~Hz}$. In the B-LSSM, parameters $g_{B}, t b$ and $g_{Y B}$ affect the GW signals within the regions: $0.49<g_{B}<0.8,19<t b<31$ and $-0.21<g_{Y B}<-0.1$. Besides, we can easily find that parameters $m_{\eta}, m_{\bar{\eta}}, \mu_{\eta}$ and $B_{\eta}$ fluctuate within small regions and exist relationship: $\frac{B_{\eta}}{\sqrt{\left(m_{\eta}^{2}+\mu_{\eta}^{2}\right)\left(m_{\bar{\eta}}^{2}+\mu_{\eta}^{2}\right)}} \simeq 1.25$. Therefore, the parameter regions in the B-LSSM are very fine-tuned to obtain the strength parameter $\alpha_{\theta} \sim 0.14$ and the ratio of speed to the Hubble rate $\beta / H_{n} \sim 5$, and then obtain observable GW signals. Then, we research the total contributions for the relationship of parameters $m_{\eta}, m_{\bar{\eta}}, \mu_{\eta}$ and $B_{\eta}$ after considering the one-loop correction through the oneloop zero temperature effective potential with the CP-even Higgs components $\phi_{\eta}$ and $\phi_{\bar{\eta}}$ : $A_{\text {one-loop }} \equiv \frac{B_{\eta}+\Delta_{B_{\eta}}}{\sqrt{\left(m_{\eta}^{2}+\mu_{\eta}^{2}+\Delta_{11}\right)\left(m_{\bar{\eta}}^{2}+\mu_{\eta}^{2}+\Delta_{22}\right)}}$. The contribution of one-loop corrections are small and only around $2 \% \sim 6 \%$ of the total contributions. As the parameters take the suitable values, the strength of the GW spectrum will be as large as $h^{2} \Omega_{G W} \sim 10^{-9}$, which may be detected by the future GW detection experiments such as LISA, BBO, DECIGO and Ultimate-DECIGO. 


\section{Acknowledgments}

We are very grateful to Li-Gong Bian the teacher of Chongqing University and Wu-Long $\mathrm{Xu}$ the Dr. of Beijing University of Technology, for giving us some useful discussions. This work is supported by the Major Project of National Natural Science Foundation of China (NNSFC) No. 12075074, No. 11535002 and No. 11705045, Natural Science Foundation of Hebei Province No. A2020201002 and the youth top-notch talent support program of the Hebei Province.

\section{A The anomaly free of B-LSSM}

B-LSSM is assumed the gauged one, and we have proved carefully that this model is anomaly free. We use $Y^{Y}$ and $Y^{B-L}$ respectively representing the charge of $\mathrm{U}(1)_{Y}$ and $\mathrm{U}(1)_{B-L}$. The quantum field theory written by M. E. Peskin has introduced the anomaly free of standard model(SM) in page 705-707. So, we show the demonstration of anomaly free in the B-LSSM.

1. In the B-LSSM, the anomaly of three SU(2) bosons banishes, as well as the anomaly of three $\mathrm{SU}(3)$ boson. These anomalies are same as the SM.

2. In the B-LSSM, the anomalies containing one $\mathrm{SU}(3)$ boson or one $\mathrm{SU}(2)$ boson are proportional to $\operatorname{Tr}\left[t^{a}\right]=0$ or $\operatorname{Tr}\left[\tau^{a}\right]=0$.

3. The remaining nontrivial anomalies are: the anomaly of one $\mathrm{U}(1)$ boson with two $\mathrm{SU}(3)$ bosons, the anomaly of one $\mathrm{U}(1)$ boson with two $\mathrm{SU}(2)$ bosons, the anomaly of three $\mathrm{U}(1)$ bosons and the gravitational anomaly with one $\mathrm{U}(1)$ boson. In the B-LSSM, there are $\mathrm{U}(1)_{Y}$ and $\mathrm{U}(1)_{B-L}$ gauge groups, which are more complicated than the SM.

(1) The anomaly of one $\mathrm{U}(1)_{Y}$ or $\mathrm{U}(1)_{B-L}$ boson with two $\mathrm{SU}(3)$ bosons is proportional to the group theory factor $\operatorname{Tr}\left[t^{a} t^{b} Y^{Y}\right]=\frac{1}{2} \delta^{a b} \sum_{q} Y_{q}^{Y}$ or $\operatorname{Tr}\left[t^{a} t^{b} Y^{B-L}\right]=\frac{1}{2} \delta^{a b} \sum_{q} Y_{q}^{B-L}$.

(2) Similarly, the anomaly of one $\mathrm{U}(1)_{Y}$ or $\mathrm{U}(1)_{B-L}$ boson with two $\mathrm{SU}(2)$ bosons is also proportional to the group theory factor $\operatorname{Tr}\left[\tau^{a} \tau^{b} Y^{Y}\right]=\frac{1}{2} \delta^{a b} \sum_{f L} Y_{f L}^{Y}$ or $\operatorname{Tr}\left[\tau^{a} \tau^{b} Y^{B-L}\right]=\frac{1}{2} \delta^{a b} \sum_{f L} Y_{f L}^{B-L}$.

(3) The anomalies of three U(1) gauge bosons are divided into four types with the B-LSSM: $\operatorname{Tr}\left[Y^{Y} Y^{Y} Y^{Y}\right]=\sum_{n}\left(Y_{n}^{Y}\right)^{3}, \operatorname{Tr}\left[Y^{B-L} Y^{B-L} Y^{B-L}\right]=\sum_{n}\left(Y_{n}^{B-L}\right)^{3}$, $\operatorname{Tr}\left[Y^{Y} Y^{B-L} Y^{B-L}\right]=\sum_{n}\left(Y_{n}^{Y}\right)\left(Y_{n}^{B-L}\right)^{2}$ and $\operatorname{Tr}\left[Y^{Y} Y^{Y} Y^{B-L}\right]=\sum_{n}\left(Y_{n}^{Y}\right)^{2}\left(Y_{n}^{B-L}\right)$.

(4) The gravitational anomaly with one $\mathrm{U}(1)$ gauge boson $\mathrm{U}(1)_{Y}$ or $\mathrm{U}(1)_{B-L}$ is proportional to $\operatorname{Tr}\left[Y^{Y}\right]=\sum_{n}\left(Y_{n}^{Y}\right)$ or $\operatorname{Tr}\left[Y^{B-L}\right]=\sum_{n}\left(Y_{n}^{B-L}\right)$. 
The anomalies that do not relate with $\mathrm{U}(1)_{B-L}$ are proved free and they are very similar as the SM condition.

$$
\begin{aligned}
\sum_{q} Y_{q}^{Y}= & {\left[\frac{1}{6} \times 2+\frac{1}{3}+\left(-\frac{2}{3}\right)\right] \times 3 \times 3=0, } \\
\sum_{f L} Y_{f L}^{Y}= & \frac{1}{6} \times 3 \times 3+\left(-\frac{1}{2}\right) \times 3+\frac{1}{2}+\left(-\frac{1}{2}\right)=0, \\
\sum_{n}\left(Y_{n}^{Y}\right)^{3}= & {\left[\left(\frac{1}{6}\right)^{3} \times 2+\left(\frac{1}{3}\right)^{3}+\left(-\frac{2}{3}\right)^{3}\right] \times 3 \times 3+\left[\left(-\frac{1}{2}\right)^{3} \times 2+1^{3}\right] \times 3 } \\
& +\left(\frac{1}{2}\right)^{3} \times 2+\left(-\frac{1}{2}\right)^{3} \times 2=0, \\
\sum_{n} Y_{n}^{Y}= & {\left[\frac{1}{6} \times 2+\frac{1}{3}+\left(-\frac{2}{3}\right)\right] \times 3 \times 3+\left[\left(-\frac{1}{2}\right) \times 2+1\right] \times 3+\left(\frac{1}{2}\right) \times 2+\left(-\frac{1}{2}\right) \times 2=0 . }
\end{aligned}
$$

The anomalies including $\mathrm{U}(1)_{B-L}$ are also proved free, which are more complicated than the calculation of SM.

$$
\begin{aligned}
\sum_{q} Y_{q}^{B-L}= & {\left[\frac{1}{6} \times 2+\left(-\frac{1}{6}\right)+\left(-\frac{1}{6}\right)\right] \times 3 \times 3=0 } \\
\sum_{f L} Y_{f L}^{B-L}= & \frac{1}{6} \times 3 \times 3+\left(-\frac{1}{2}\right) \times 3=0 \\
\sum_{n}\left(Y_{n}^{B-L}\right)^{3}= & {\left[\left(\frac{1}{6}\right)^{3} \times 2+\left(-\frac{1}{6}\right)^{3}+\left(-\frac{1}{6}\right)^{3}\right] \times 3 \times 3+\left[\left(-\frac{1}{2}\right)^{3} \times 2+\left(\frac{1}{2}\right)^{3}+\left(\frac{1}{2}\right)^{3}\right] \times 3 } \\
& +1^{3}+(-1)^{3}=0 \\
\sum_{n} Y_{n}^{Y}\left(Y_{n}^{B-L}\right)^{2}= & {\left[\frac{1}{6} \times\left(\frac{1}{6}\right)^{2} \times 2+\frac{1}{3} \times\left(-\frac{1}{6}\right)^{2}+\left(-\frac{2}{3}\right)^{2} \times\left(-\frac{1}{6}\right)\right] \times 3 \times 3 } \\
& +\left[\left(-\frac{1}{2}\right)^{2} \times\left(-\frac{1}{2}\right) \times 2+1 \times\left(\frac{1}{2}\right)^{2}\right] \times 3=0, \\
& +\left[\left(-\frac{1}{2}\right) \times\left(-\frac{1}{2}\right)^{2} \times 2+1^{2} \times \frac{1}{2}\right] \times 3=0, \\
\sum_{n}\left(Y_{n}^{Y}\right)^{2}\left(Y_{n}^{B-L}\right)= & {\left[\left(\frac{1}{6} \times 2+\left(\frac{1}{3}\right)^{2} \times\left(-\frac{1}{6}\right)+\left(-\frac{2}{3}\right) \times\left(-\frac{1}{6}\right)^{2}\right] \times 3 \times 3\right.} \\
\sum_{n} Y_{n}^{B-L}= & {\left[\frac{1}{6} \times 2+\left(-\frac{1}{6}\right)+\left(-\frac{1}{6}\right)\right] \times 3 \times 3+\left[\left(-\frac{1}{2}\right) \times 2+\frac{1}{2}+\frac{1}{2}\right] \times 3+1+(-1)=0 . }
\end{aligned}
$$

\section{B The vacuum of Higgs doublets and Higgs singlets}

In the B-LSSM, the Higgs doublets and Higgs singlets obtain the nonzero vacuum expectation values(VEVs), which break the gauge group $\mathrm{SU}(3) \otimes \mathrm{U}(1)_{Y} \otimes \mathrm{U}(1)_{B-L}$ down to $\mathrm{U}(1)_{e m}$ at zero temperature. For the model's parameter values considered in our analysis, the vacuum of eq.2 is indeed the global one and there is no charge breaking nor CP violating deeper minima. 
First, in the MSSM, there are two Higgs doublets $H_{d}$ and $H_{u}$. So the tree-level effective potential can be written as $V_{M S S M}=\frac{1}{8}\left(g_{1}^{2}+g_{2}^{2}\right)\left(\left|H_{u}^{0}\right|^{2}+\left|H_{u}^{+}\right|^{2}-\left|H_{d}^{0}\right|^{2}-\left|H_{d}^{-}\right|^{2}\right)^{2}+$ $\frac{1}{2} g_{2}^{2}\left|H_{u}^{0} H_{d}^{-*}+H_{u}^{+} H_{d}^{0 *}\right|^{2}+\left(|\mu|^{2}+m_{H_{u}}^{2}\right)\left(\left|H_{u}^{0}\right|^{2}+\left|H_{u}^{+}\right|^{2}\right)+\left(|\mu|^{2}+m_{H_{d}}^{2}\right)\left(\left|H_{d}^{0}\right|^{2}+\left|H_{d}^{-}\right|^{2}\right)+$ $\left(B_{\mu}\left(H_{u}^{+} H_{d}^{-}-H_{u}^{0} H_{d}^{0}\right)+\right.$ H.c. $)$. On the premise of not losing generality, the potential energy minimum point $H_{u}^{+}=0$ is selected, and $H_{d}^{-}=0$ is required. Therefore, the charged component of the Higgs scalar potential cannot obtain the vacuum expected value, and the electromagnetic action at the potential energy minimum point must not be broken. When $H_{u}^{+}=H_{d}^{-}=0$, the scalar potential of MSSM is $V_{M S S M}^{\prime}=\frac{1}{8}\left(g_{1}^{2}+g_{2}^{2}\right)\left(\left|H_{u}^{0}\right|^{2}-\left|H_{d}^{0}\right|^{2}\right)^{2}+$ $\left(|\mu|^{2}+m_{H_{u}}^{2}\right)\left|H_{u}^{0}\right|^{2}+\left(|\mu|^{2}+m_{H_{d}}^{2}\right)\left|H_{d}^{0}\right|^{2}+\left(-B_{\mu} H_{u}^{0} H_{d}^{0}+\right.$ H.c. $)$. Only the parameter $B_{\mu}$ in this effective potential depends on the field phase. The phase of $H_{u}^{0}$ and $H_{d}^{0}$ will be defined to absorb the phase of $B_{\mu}$, which takes to be real and positive. The minimum point of the effective potential requires that $H_{u}^{0} H_{d}^{0}$ is also real and positive, so that $\left\langle H_{d}^{0}\right\rangle$ and $<H_{u}^{0}>$ must have opposite phases. $H_{u}$ and $H_{d}$ have opposite weak supercharges $\left( \pm \frac{1}{2}\right)$, so the $\mathrm{U}(1)_{Y}$ gauge transformation is used to make $<H_{d}^{0}>$ and $<H_{u}^{0}>$ real and positive without losing generality. The convention that the vacuum expectation values and $B_{\mu}$ are selected as real at the same time leads to the spontaneous breaking of Higgs scalar potential in MSSM without CP violating. In order to make the scalar potential feasible, it is necessary to satisfy: $2 B_{\mu}<2|\mu|^{2}+m_{H_{u}}^{2}+m_{H_{d}}^{2}$ and $B_{\mu}^{2}>\left(|\mu|^{2}+m_{H_{u}}^{2}\right)\left(|\mu|^{2}+m_{H_{d}}^{2}\right)$. At this time, there exists electroweak symmetry spontaneous breaking and the corresponding vacuum expectation values are obtained.

In the B-LSSM, $\mathrm{U}(1)_{B-L}$ gauge group will be added to the MSSM gauge group, and two singlet Higgs fields $\eta$ and $\bar{\eta}$ will be added. Therefore, in the B-LSSM, the tree-level scalar potential is written as $V_{B-L}=V_{M S S M}^{\prime}+\frac{1}{8} g_{Y B}^{2}\left(\left|H_{u}^{0}\right|^{2}-\left|H_{d}^{0}\right|^{2}\right)^{2}+\frac{1}{2} g_{B} g_{Y B}\left(\left|H_{u}^{0}\right|^{2}|\bar{\eta}|^{2}+\right.$ $\left.\left|H_{d}^{0}\right|^{2}|\eta|^{2}-\left|H_{u}^{0}\right|^{2}|\eta|^{2}-\left|H_{d}^{0}\right|^{2}|\bar{\eta}|^{2}\right)+\frac{1}{2} g_{B}^{2}\left(|\eta|^{2}-|\bar{\eta}|^{2}\right)^{2}+\left(\left|\mu_{\eta}\right|^{2}+m_{\eta}^{2}\right)|\eta|^{2}+\left(\left|\mu_{\eta}\right|^{2}+m_{\bar{\eta}}^{2}\right)|\bar{\eta}|^{2}+$ $\left(-B_{\eta} \eta \bar{\eta}+\right.$ H.c.). The parameter $B_{\eta}$ in the effective potential also depends on the field phase. The phase of $\eta$ and $\bar{\eta}$ will be defined to absorb the phase of $B_{\eta}$, which takes as real and positive. The minimum point of the effective potential requires that $\eta \bar{\eta}$ is also real and positive, so that $\langle\eta\rangle$ and $\langle\bar{\eta}\rangle$ must have opposite phases. $\eta$ and $\bar{\eta}$ have opposite weak supercharges $( \pm 1)$, so the $\mathrm{U}(1)_{B-L}$ gauge transformation is used to make $\eta$ and $\bar{\eta}$ real and positive without losing generality. The convention that the vacuum expectation values and $B_{\eta}$ are selected as real simultaneously causes the spontaneous breaking of Higgs scalar potential in the B-LSSM without CP violating. In order to make the scalar potential feasible, it also requires that: $2 B_{\eta}<2\left|\mu_{\eta}\right|^{2}+m_{\eta}^{2}+m_{\bar{\eta}}^{2}$ and $B_{\eta}^{2}>\left(\left|\mu_{\eta}\right|^{2}+m_{\eta}^{2}\right)\left(|\mu|^{2}+m_{\bar{\eta}}^{2}\right)$. At this time, there exists electroweak symmetry spontaneous breaking and the corresponding vacuum expectation values are obtained in the B-LSSM. The parameter space we chosen satisfies these conditions.

\section{The value of parameter $g_{Y B}$}

The range $[-0.45,-0.05]$ assumed for $g_{Y B}$ applies to the total contribution including oneloop effects from fields carrying both $\mathrm{Y}$ and B-L charges. The gauge groups $\mathrm{U}(1)_{Y}$ and $\mathrm{U}(1)_{B-L}$ mix together, and the coupling matrix is: $\left(\begin{array}{cc}g_{Y Y} & g_{Y B}^{\prime} \\ g_{B Y}^{\prime} & g_{B B}\end{array}\right)$. Through the orthogo- 
nal matrix $R$, the coupling matrix can be rewritten as: $\left(\begin{array}{cc}g_{Y Y} & g_{Y B}^{\prime} \\ g_{B Y}^{\prime} & g_{B B}\end{array}\right) R=\left(\begin{array}{cc}g_{1} & g_{Y B} \\ 0 & g_{B}\end{array}\right)$. Then, we can redefine the $\mathrm{U}(1)$ gauge fields: $R^{T}\left(\begin{array}{c}B_{\mu}^{\prime Y} \\ B_{\mu}^{\prime B L}\end{array}\right)=\left(\begin{array}{c}B_{\mu} \\ B_{\mu}^{\prime \prime}\end{array}\right)$. In ref. [58], the $\beta$-functions of eqs. (B.16) and (B.17) are respectively $\beta_{g_{Y} B}^{(1)}=\frac{3}{5}\left[g_{Y Y} g_{B Y}^{\prime}\left(15 g_{B B}+\right.\right.$ $\left.\left.2 \sqrt{10} g_{Y B}^{\prime}\right)+g_{Y Y}^{2}\left(11 g_{Y B}^{\prime}+2 \sqrt{10} g_{B B}\right)+g_{Y B}^{\prime}\left(11 g_{Y B}^{\prime 2}+15 g_{B B}^{2}+4 \sqrt{10} g_{Y B}^{\prime} g_{B B}\right)\right]$ and $\beta_{g_{B Y}}^{(1)}=$ $\frac{3}{5}\left[11 g_{Y Y}^{2} g_{B Y}^{\prime}+g_{Y Y}\left(11 g_{Y B}^{\prime} g_{B B}+2 \sqrt{10}\left(2 g_{B Y}^{\prime 2}+g_{B B}^{2}\right)\right)+g_{B Y}^{\prime}\left(15\left(g_{B B}^{2}+g_{B Y}^{\prime 2}\right)+2 \sqrt{10} g_{Y B}^{\prime} g_{B B}\right)\right]$. For vanishing off-diagonal gauge couplings, the $\beta$-functions of eqs. (B.16) and (B.17) will always be positive. As parameters $g_{Y Y}$ and $g_{B B}$ are positive, parameters $g_{Y B}^{\prime}$ and $g_{B Y}^{\prime}$ can be driven negative. Besides, at the electroweak scale, $g_{Y B}=\frac{g_{Y B}^{\prime} g_{B B}+g_{B Y}^{\prime} g_{Y Y}}{\sqrt{g_{B B}^{2}+g_{B Y}^{\prime}}}$, which indicates that parameter $g_{Y B}$ is driven negative. In ref. [59], the author also indicates that $g_{Y B} \simeq-0.147$ at SUSY scale. Therefore, the value of parameter $g_{Y B}$ will be taken around $[-0.45,-0.05]$ in our numerical calculation.

\section{The neutralino mass matrix}

In the basis $\left(\lambda_{Y}, \lambda_{W}^{3}, \psi_{H_{d}}^{1}, \psi_{H_{u}}^{2}, \lambda_{B^{\prime}}, \psi_{\eta}, \psi_{\bar{\eta}}\right)$, we can obtain a $7 \times 7$ neutralino mass matrix:

$$
M_{\chi^{0}}=\left(\begin{array}{ccccccc}
M_{1} & 0 & -\frac{1}{2} g_{1} v_{d} & \frac{1}{2} g_{1} v_{u} & M_{B B^{\prime}} & 0 & 0 \\
0 & M_{2} & \frac{1}{2} g_{2} v_{d} & -\frac{1}{2} g_{2} v_{u} & 0 & 0 & 0 \\
-\frac{1}{2} g_{1} v_{d} & \frac{1}{2} g_{2} v_{d} & 0 & -\mu & -\frac{1}{2} g_{Y B} v_{d} & 0 & 0 \\
\frac{1}{2} g_{1} v_{u} & -\frac{1}{2} g_{2} v_{u} & -\mu & 0 & \frac{1}{2} g_{Y B} v_{u} & 0 & 0 \\
M_{B B^{\prime}} & 0 & -\frac{1}{2} g_{Y B} v_{d} & \frac{1}{2} g_{Y B} v_{u} & M_{B L} & -g_{B} v_{\eta} g_{B} v_{\bar{\eta}} \\
0 & 0 & 0 & 0 & -g_{B} v_{\eta} & 0 & -\mu_{\eta} \\
0 & 0 & 0 & 0 & g_{B} v_{\bar{\eta}} & -\mu_{\eta} & 0
\end{array}\right)
$$

The value of $\mathrm{U}(1)_{B-L}$ breaking Higgs singlets VEV $u$ is around $u \simeq 10 \mathrm{TeV}$, which is much larger than the Higgs doublets VEV $v \simeq 246 \mathrm{GeV}$. Besides, we only consider the CP-even Higgs components $\phi_{\eta}$ and $\phi_{\bar{\eta}}$ to study the strong first-order PT, so the components from $\mathrm{SU}(2)_{L} \times \mathrm{U}(1)_{Y}$ gauginos are not included.

\section{E The one-loop correction $A_{\text {one-loop }}$}

We consider the one-loop correction through the one-loop zero temperature effective potential with the CP-even Higgs components $\phi_{\eta}$ and $\phi_{\bar{\eta}}$ : $A_{\text {one-loop }} \equiv$ $\frac{B_{\eta}+\Delta_{B_{\eta}}}{\sqrt{\left(m_{\eta}^{2}+\mu_{\eta}^{2}+\Delta_{11}\right)\left(m_{\bar{\eta}}^{2}+\mu_{\eta}^{2}+\Delta_{22}\right)}}$. The corresponding theoretical analyses will be given out as follows: $\Delta_{11}=\left[-\frac{1}{\phi_{\eta}} \frac{\partial \Delta V_{1}}{\partial \phi_{\eta}}+\frac{\partial^{2} \Delta V_{1}}{\partial \phi_{\eta}^{2}}\right]_{\phi_{\eta}=v_{\eta}}, \Delta_{22}=\left[-\frac{1}{\phi_{\bar{\eta}}} \frac{\partial \Delta V_{1}}{\partial \phi_{\bar{\eta}}}+\frac{\partial^{2} \Delta V_{1}}{\partial \phi_{\bar{\eta}}^{2}}\right]_{\phi_{\bar{\eta}}=v_{\bar{\eta}}}$ and $\Delta_{B_{\eta}}=$ $\left[\frac{\partial^{2} \Delta V_{1}}{\partial \phi_{\eta} \partial \phi_{\bar{\eta}}}\right]_{\phi_{\eta}=v_{\eta}, \phi_{\bar{\eta}}=v_{\bar{\eta}}} . \Delta V_{1}$ represents the one-loop zero temperature effective potential:

$$
\Delta V_{1}\left(\phi_{\eta}, \phi_{\bar{\eta}}, 0\right)=\sum_{i}(-1)^{k} \frac{n_{i}}{64 \pi^{2}} m_{i}^{4}\left(\phi_{\eta}, \phi_{\bar{\eta}}\right)\left[\log \frac{m_{i}^{2}\left(\phi_{\eta}, \phi_{\bar{\eta}}\right)}{\Lambda^{2}}-c_{i}\right] .
$$


Therefore, the radiative corrections $\Delta_{i i}$ from the one-loop zero temperature effective potential will be deduced as:

$$
\begin{aligned}
& \Delta_{i i}=\frac{n_{i}}{(4 \pi)^{2}}[ \sum_{k=\chi_{i}^{0}}\left(\frac{2}{\phi_{i}} f\left(m_{k}^{2}\right) \frac{\partial m_{k}^{2}}{\partial \phi_{i}}-2 f\left(m_{k}^{2}\right) \frac{\partial^{2} m_{k}^{2}}{\partial \phi_{i}^{2}}-\frac{1}{2} \ln \left(\frac{m_{k}^{2}}{\Lambda^{2}}\right) \frac{\partial m_{k}^{2}}{\partial \phi_{i}} \frac{\partial m_{k}^{2}}{\partial \phi_{i}}\right) \\
&\left.+\sum_{k=\tilde{S}}\left(-\frac{1}{\phi_{i}} f\left(m_{k}^{2}\right) \frac{\partial m_{k}^{2}}{\partial \phi_{i}}+f\left(m_{k}^{2}\right) \frac{\partial^{2} m_{k}^{2}}{\partial \phi_{i}^{2}}+\frac{1}{4} \ln \left(\frac{m_{k}^{2}}{\Lambda^{2}}\right) \frac{\partial m_{k}^{2}}{\partial \phi_{i}} \frac{\partial m_{k}^{2}}{\partial \phi_{i}}\right)\right], \\
& \Delta_{i j}=\frac{n_{i}}{(4 \pi)^{2}} \sum_{k=\phi_{\eta}, \phi_{\bar{\eta}}, \sigma_{\eta}, \sigma_{\bar{\eta}}}\left(f\left(m_{k}^{2}\right) \frac{\partial^{2} m_{k}^{2}}{\partial \phi_{i} \partial \phi_{j}}+\frac{1}{4} \ln \left(\frac{m_{k}^{2}}{\Lambda^{2}}\right) \frac{\partial m_{k}^{2}}{\partial \phi_{i}} \frac{\partial m_{k}^{2}}{\partial \phi_{j}}\right) .
\end{aligned}
$$

Here, $\phi_{i, j}$ are the CP-even Higgs components $\phi_{\eta}, \quad \phi_{\bar{\eta}}, \quad \tilde{S} \in$ $\left(Z^{\prime}, \tilde{Q}^{I}, \tilde{U}^{I}, \tilde{D}^{I}, \tilde{L}^{I}, \tilde{R}^{I}, \tilde{\nu}_{i}, \tilde{\nu}_{i}^{R}, \phi_{\eta}, \phi_{\bar{\eta}}, \sigma_{\eta}, \sigma_{\bar{\eta}}\right) . \quad f\left(m_{k}^{2}\right)=\frac{1}{4} m_{k}^{2}\left[\ln \left(\frac{m_{k}^{2}}{\Lambda^{2}}\right)-1\right], \Lambda$ is the renormalization scale and we take $\Lambda=1 \mathrm{TeV}$ in the numerical discussion. $m_{k}$ represent the corresponding particle masses.

Open Access. This article is distributed under the terms of the Creative Commons Attribution License (CC-BY 4.0), which permits any use, distribution and reproduction in any medium, provided the original author(s) and source are credited.

\section{References}

[1] H.P. Nilles, Supersymmetry, supergravity and particle physics, Phys. Rept. 110 (1984) 1 [INSPIRE].

[2] H.E. Haber and G.L. Kane, The search for supersymmetry: probing physics beyond the standard model, Phys. Rept. 117 (1985) 75 [INSPIRE].

[3] J. Rosiek, Complete set of Feynman rules for the minimal supersymmetric extension of the standard model, Phys. Rev. D 41 (1990) 3464 [INSPIRE].

[4] T.-F. Feng and X.-Y. Yang, Renormalization and two loop electroweak corrections to lepton anomalous dipole moments in the standard model and beyond (I): heavy fermion contributions, Nucl. Phys. B 814 (2009) 101 [arXiv:0901.1686] [INSPIRE].

[5] V. Barger, P. Fileviez Perez and S. Spinner, Minimal gauged $\mathrm{U}(1)_{B-L}$ model with spontaneous R-parity violation, Phys. Rev. Lett. 102 (2009) 181802 [arXiv:0812.3661] [INSPIRE].

[6] P. Fileviez Perez and S. Spinner, Spontaneous R-parity breaking and left-right symmetry, Phys. Lett. B 673 (2009) 251 [arXiv:0811.3424] [INSPIRE].

[7] M. Ambroso and B.A. Ovrut, The mass spectra, hierarchy and cosmology of B-L MSSM heterotic compactifications, Int. J. Mod. Phys. A 26 (2011) 1569 [arXiv:1005.5392] [INSPIRE].

[8] P. Fileviez Perez and S. Spinner, The fate of R-parity, Phys. Rev. D 83 (2011) 035004 [arXiv: 1005.4930] [INSPIRE].

[9] J.-L. Yang, T.-F. Feng, S.-M. Zhao, R.-F. Zhu, X.-Y. Yang and H.-B. Zhang, Two loop electroweak corrections to $\bar{B} \rightarrow X_{s} \gamma$ and $B_{s}^{0} \rightarrow \mu^{+} \mu^{-}$in the B-LSSM,

Eur. Phys. J. C 78 (2018) 714 [arXiv:1803.09904] [INSPIRE]. 
[10] C.S. Aulakh, A. Melfo, A. Rasin and G. Senjanović, Seesaw and supersymmetry or exact R-parity, Phys. Lett. B 459 (1999) 557 [hep-ph/9902409] [INSPIRE].

[11] W. Abdallah, A. Hammad, S. Khalil and S. Moretti, Search for mono-Higgs signals at the LHC in the B-L supersymmetric standard model, Phys. Rev. D 95 (2017) 055019 [arXiv: 1608.07500] [INSPIRE].

[12] J.-L. Yang, T.-F. Feng and H.-B. Zhang, Electroweak baryogenesis and electron EDM in the B-LSSM, Eur. Phys. J. C 80 (2020) 210 [arXiv: 2002.09313] [InSPIRE].

[13] S. Khalil and H. Okada, Dark matter in B-L extended MSSM models, Phys. Rev. D 79 (2009) 083510 [arXiv:0810.4573] [InSPIRE].

[14] L. Basso, B. O'Leary, W. Porod and F. Staub, Dark matter scenarios in the minimal SUSY B-L model, JHEP 09 (2012) 054 [arXiv:1207.0507] [INSPIRE].

[15] L. Delle Rose, S. Khalil, S.J.D. King, C. Marzo, S. Moretti and C.S. Un, Naturalness and dark matter in the supersymmetric B-L extension of the standard model, Phys. Rev. D 96 (2017) 055004 [arXiv:1702.01808] [InSPIRE].

[16] L. Delle Rose et al., Sneutrino dark matter in the BLSSM, JHEP 07 (2018) 100 [arXiv: 1712.05232] [INSPIRE].

[17] LigO ScIentific and ViRgo collaborations, Observation of gravitational waves from a binary black hole merger, Phys. Rev. Lett. 116 (2016) 061102 [arXiv:1602. 03837] [INSPIRE].

[18] LigO Scientific and Virgo collaborations, Binary black hole mergers in the first advanced LIGO observing run, Phys. Rev. X 6 (2016) 041015 [Erratum ibid. 8 (2018) 039903] [arXiv: 1606.04856] [INSPIRE].

[19] E. Witten, Cosmic separation of phases, Phys. Rev. D 30 (1984) 272 [InSPIRE].

[20] C.J. Hogan, Gravitational radiation from cosmological phase transitions, Mon. Not. Roy. Astron. Soc. 218 (1986) 629 [INSPIRE].

[21] A. Kosowsky, M.S. Turner and R. Watkins, Gravitational radiation from colliding vacuum bubbles, Phys. Rev. D 45 (1992) 4514 [InSPIRE].

[22] M. Kamionkowski, A. Kosowsky and M.S. Turner, Gravitational radiation from first order phase transitions, Phys. Rev. D 49 (1994) 2837 [astro-ph/9310044] [INSPIRE].

[23] R.-G. Cai, Z. Cao, Z.-K. Guo, S.-J. Wang and T. Yang, The gravitational-wave physics, Natl. Sci. Rev. 4 (2017) 687 [arXiv: 1703.00187] [INSPIRE].

[24] C. Caprini et al., Science with the space-based interferometer eLISA. Part II. Gravitational waves from cosmological phase transitions, JCAP 04 (2016) 001 [arXiv:1512.06239] [INSPIRE].

[25] W. Chao, W.-F. Cui, H.-K. Guo and J. Shu, Gravitational wave imprint of new symmetry breaking, Chin. Phys. C 44 (2020) 123102 [arXiv:1707.09759] [INSPIRE].

[26] K. Kajantie, K. Rummukainen and M.E. Shaposhnikov, A lattice Monte Carlo study of the hot electroweak phase transition, Nucl. Phys. B 407 (1993) 356 [hep-ph/9305345] [INSPIRE].

[27] Z. Fodor, J. Hein, K. Jansen, A. Jaster and I. Montvay, Simulating the electroweak phase transition in the SU(2) Higgs model, Nucl. Phys. B 439 (1995) 147 [hep-lat/9409017] [INSPIRE]. 
[28] K. Kajantie, M. Laine, K. Rummukainen and M.E. Shaposhnikov, The electroweak phase transition: a nonperturbative analysis, Nucl. Phys. B 466 (1996) 189 [hep-lat/9510020] [INSPIRE].

[29] C. Delaunay, C. Grojean and J.D. Wells, Dynamics of non-renormalizable electroweak symmetry breaking, JHEP 04 (2008) 029 [arXiv:0711.2511] [INSPIRE].

[30] F.P. Huang, Y. Wan, D.-G. Wang, Y.-F. Cai and X. Zhang, Hearing the echoes of electroweak baryogenesis with gravitational wave detectors, Phys. Rev. D 94 (2016) 041702 [arXiv: 1601.01640] [INSPIRE].

[31] R.-G. Cai, M. Sasaki and S.-J. Wang, The gravitational waves from the first-order phase transition with a dimension-six operator, JCAP 08 (2017) 004 [arXiv:1707.03001] [INSPIRE].

[32] R. Apreda, M. Maggiore, A. Nicolis and A. Riotto, Gravitational waves from electroweak phase transitions, Nucl. Phys. B 631 (2002) 342 [gr-qc/0107033] [inSPIRE].

[33] S.J. Huber and T. Konstandin, Production of gravitational waves in the NMSSM, JCAP 05 (2008) 017 [arXiv:0709.2091] [INSPIRE].

[34] S.J. Huber, T. Konstandin, G. Nardini and I. Rues, Detectable gravitational waves from very strong phase transitions in the general NMSSM, JCAP 03 (2016) 036 [arXiv:1512.06357] [INSPIRE].

[35] F.P. Huang and X. Zhang, Probing the gauge symmetry breaking of the early universe in 3-3-1 models and beyond by gravitational waves, Phys. Lett. B 788 (2019) 288 [arXiv: 1701.04338] [INSPIRE].

[36] W. Chao, H.-K. Guo and J. Shu, Gravitational wave signals of electroweak phase transition triggered by dark matter, JCAP 09 (2017) 009 [arXiv: 1702.02698] [INSPIRE].

[37] A. Addazi and A. Marciano, Limiting majoron self-interactions from gravitational wave experiments, Chin. Phys. C 42 (2018) 023105 [arXiv: 1705. 08346] [INSPIRE].

[38] LISA collaboration, Laser Interferometer Space Antenna, arXiv:1702.00786 [INSPIRE].

[39] H. Kudoh, A. Taruya, T. Hiramatsu and Y. Himemoto, Detecting a gravitational-wave background with next-generation space interferometers, Phys. Rev. D 73 (2006) 064006 [gr-qc/0511145] [INSPIRE].

[40] A.D. Sakharov, Violation of $C P$ invariance, $C$ asymmetry, and baryon asymmetry of the universe, Pisma Zh. Eksp. Teor. Fiz. 5 (1967) 32 [JETP Lett. 5 (1967) 24] [Sov. Phys. Usp. 34 (1991) 392] [Usp. Fiz. Nauk 161 (1991) 61] [INSPIRE].

[41] S. Bruggisser, T. Konstandin and G. Servant, CP-violation for electroweak baryogenesis from dynamical CKM matrix, JCAP 11 (2017) 034 [arXiv: 1706. 08534] [INSPIRE].

[42] D.E. Morrissey and M.J. Ramsey-Musolf, Electroweak baryogenesis, New J. Phys. 14 (2012) 125003 [arXiv: 1206 . 2942] [INSPIRE].

[43] A.I. Bochkarev and M.E. Shaposhnikov, Electroweak production of baryon asymmetry and upper bounds on the Higgs and top masses, Mod. Phys. Lett. A 2 (1987) 417 [InSPIRE].

[44] F. Csikor, Z. Fodor and J. Heitger, Endpoint of the hot electroweak phase transition, Phys. Rev. Lett. 82 (1999) 21 [hep-ph/9809291] [INSPIRE]. 
[45] LEP Working Group for Higgs boson searches, ALEPH, DELPHI, L3 and OPAL collaborations, Search for the standard model Higgs boson at LEP,

Phys. Lett. B 565 (2003) 61 [hep-ex/0306033] [INSPIRE].

[46] ATLAS collaboration, Observation of a new particle in the search for the standard model Higgs boson with the ATLAS detector at the LHC, Phys. Lett. B 716 (2012) 1 [arXiv: 1207.7214] [INSPIRE].

[47] CMS collaboration, Observation of a new boson at a mass of $125 \mathrm{GeV}$ with the CMS experiment at the LHC, Phys. Lett. B $\mathbf{7 1 6}$ (2012) 30 [arXiv:1207.7235] [INSPIRE].

[48] J.E. Camargo-Molina, B. O'Leary, W. Porod and F. Staub, Stability of R-parity in supersymmetric models extended by $\mathrm{U}(1)_{B-L}$, Phys. Rev. D 88 (2013) 015033 [arXiv: 1212.4146] [INSPIRE].

[49] Particle Data Group collaboration, Review of particle physics, PTEP 2020 (2020) $083 \mathrm{C} 01$ [INSPIRE].

[50] ATLAS collaboration, Search for bottom-squark pair production with the ATLAS detector in final states containing Higgs bosons, b-jets and missing transverse momentum, JHEP 12 (2019) 060 [arXiv: 1908.03122] [INSPIRE].

[51] ATLAS collaboration, Search for top squarks in events with a Higgs or Z boson using $139 \mathrm{fb}^{-1}$ of $\mathrm{pp}$ collision data at $\sqrt{\mathrm{s}}=13 \mathrm{TeV}$ with the ATLAS detector, Eur. Phys. J. C 80 (2020) 1080 [arXiv:2006.05880] [inSPIRE].

[52] X.-X. Dong, T.-F. Feng, S.-M. Zhao and H.-B. Zhang, The naturalness in the BLMSSM and B-LSSM, Eur. Phys. J. C 80 (2020) 1206 [arXiv: 2005.03351] [INSPIRE].

[53] ATLAS collaboration, Search for high-mass dilepton resonances using $139 \mathrm{fb}^{-1}$ of $\mathrm{pp}$ collision data collected at $\sqrt{s}=13 \mathrm{TeV}$ with the ATLAS detector, Phys. Lett. B 796 (2019) 68 [arXiv:1903.06248] [INSPIRE].

[54] G. Cacciapaglia, C. Csáki, G. Marandella and A. Strumia, The minimal set of electroweak precision parameters, Phys. Rev. D 74 (2006) 033011 [hep-ph/0604111] [INSPIRE].

[55] M. Carena, A. Daleo, B.A. Dobrescu and T.M.P. Tait, $Z^{\prime}$ gauge bosons at the Tevatron, Phys. Rev. D 70 (2004) 093009 [hep-ph/0408098] [INSPIRE].

[56] F. Mahmoudi, New constraints on supersymmetric models from $b \rightarrow s \gamma$, JHEP 12 (2007) 026 [arXiv:0710.3791] [INSPIRE].

[57] K.A. Olive and L. Velasco-Sevilla, Constraints on supersymmetric flavour models from $b \rightarrow s \gamma$, JHEP 05 (2008) 052 [arXiv: 0801.0428] [INSPIRE].

[58] B. O'Leary, W. Porod and F. Staub, Mass spectrum of the minimal SUSY B-L model, JHEP 05 (2012) 042 [arXiv: 1112.4600] [INSPIRE].

[59] L. Basso, The Higgs sector of the minimal SUSY B-L model, Adv. High Energy Phys. 2015 (2015) 980687 [arXiv:1504.05328] [INSPIRE].

[60] L. Dolan and R. Jackiw, Symmetry behavior at finite temperature, Phys. Rev. D 9 (1974) 3320 [inSPIRE].

[61] M. Quirós, Finite temperature field theory and phase transitions, in ICTP summer school in high-energy physics and cosmology, (1999), pg. 187 [hep-ph/9901312] [INSPIRE].

[62] M.E. Carrington, The effective potential at finite temperature in the standard model, Phys. Rev. D 45 (1992) 2933 [INSPIRE]. 
[63] J.R. Espinosa, M. Quirós and F. Zwirner, On the nature of the electroweak phase transition, Phys. Lett. B 314 (1993) 206 [hep-ph/9212248] [INSPIRE].

[64] D. Comelli and J.R. Espinosa, Bosonic thermal masses in supersymmetry, Phys. Rev. D 55 (1997) 6253 [hep-ph/9606438] [INSPIRE].

[65] N. Haba and T. Yamada, Gravitational waves from phase transition in minimal SUSY U(1) $B-L$ model, Phys. Rev. D 101 (2020) 075027 [arXiv:1911.01292] [INSPIRE].

[66] A.D. Linde, Fate of the false vacuum at finite temperature: theory and applications, Phys. Lett. B 100 (1981) 37 [INSPIRE].

[67] M.S. Turner, E.J. Weinberg and L.M. Widrow, Bubble nucleation in first order inflation and other cosmological phase transitions, Phys. Rev. D 46 (1992) 2384 [INSPIRE].

[68] C. Caprini et al., Detecting gravitational waves from cosmological phase transitions with LISA: an update, JCAP 03 (2020) 024 [arXiv:1910.13125] [INSPIRE].

[69] F. Giese, T. Konstandin and J. van de Vis, Model-independent energy budget of cosmological first-order phase transitions - a sound argument to go beyond the bag model, JCAP 07 (2020) 057 [arXiv: 2004.06995] [INSPIRE].

[70] J.R. Espinosa, T. Konstandin, J.M. No and G. Servant, Energy budget of cosmological first-order phase transitions, JCAP 06 (2010) 028 [arXiv: 1004.4187] [INSPIRE].

[71] M. Hindmarsh, S.J. Huber, K. Rummukainen and D.J. Weir, Numerical simulations of acoustically generated gravitational waves at a first order phase transition, Phys. Rev. D 92 (2015) 123009 [arXiv: 1504.03291] [INSPIRE].

[72] M. Hindmarsh and M. Hijazi, Gravitational waves from first order cosmological phase transitions in the sound shell model, JCAP 12 (2019) 062 [arXiv:1909.10040] [INSPIRE].

[73] M. Hindmarsh, S.J. Huber, K. Rummukainen and D.J. Weir, Shape of the acoustic gravitational wave power spectrum from a first order phase transition, Phys. Rev. D 96 (2017) 103520 [Erratum ibid. 101 (2020) 089902] [arXiv:1704.05871] [INSPIRE].

[74] S.J. Huber and T. Konstandin, Gravitational wave production by collisions: more bubbles, JCAP 09 (2008) 022 [arXiv:0806.1828] [INSPIRE].

[75] M. Hindmarsh, S.J. Huber, K. Rummukainen and D.J. Weir, Gravitational waves from the sound of a first order phase transition, Phys. Rev. Lett. 112 (2014) 041301 [arXiv: 1304.2433] [INSPIRE].

[76] C. Caprini, R. Durrer and G. Servant, The stochastic gravitational wave background from turbulence and magnetic fields generated by a first-order phase transition, JCAP 12 (2009) 024 [arXiv:0909.0622] [INSPIRE].

[77] P. Binetruy, A. Bohe, C. Caprini and J.-F. Dufaux, Cosmological backgrounds of gravitational waves and eLISA/NGO: phase transitions, cosmic strings and other sources, JCAP 06 (2012) 027 [arXiv: 1201.0983] [INSPIRE].

[78] J.-L. Yang, H.-B. Zhang, C.-X. Liu, X.-X. Dong and T.-F. Feng, Muon $(g-2)$ in the $B$-LSSM, arXiv:2104.03542 [INSPIRE].

[79] C.L. Wainwright, CosmoTransitions: computing cosmological phase transition temperatures and bubble profiles with multiple fields, Comput. Phys. Commun. 183 (2012) 2006 [arXiv:1109.4189] [INSPIRE]. 